\title{
Safety and effectiveness of acellular pertussis vaccination during pregnancy: a systematic review
}

\author{
Sabine Vygen-Bonnet ${ }^{*}$, Wiebke Hellenbrand ${ }^{1}$, Edeltraut Garbe ${ }^{2}$, Rüdiger von Kries ${ }^{3}$, Christian Bogdan ${ }^{4,5}$,
} Ulrich Heininger ${ }^{6}$, Marianne Röbl-Mathieu ${ }^{7,8}$ and Thomas Harder ${ }^{1}$

\begin{abstract}
Background: Infants $<3$ months of age are at highest risk for developing severe complications after pertussis. The majority of pregnant women has low concentrations of pertussis-specific antibodies and thus newborns are insufficiently protected by maternally transferred antibodies. Acellular pertussis vaccination during pregnancy was recently implemented in various countries. Here, we assessed the evidence for safety and effectiveness of pertussis vaccination during pregnancy.
\end{abstract}

Methods: We searched Medline, Embase, and ClinicalTrials.gov from January 1st 2010 to January 10th 2019. We assessed risk of bias (ROB) using the Cochrane ROB tool and ROBINS-I. We evaluated the quality of evidence using the GRADE approach.

Results: We identified 1273 articles and included 22 studies (14 for safety; 8 for effectiveness), comprising 1.4 million pregnant women in safety studies and 855,546 mother-infant-pairs in effectiveness studies. No significant differences between vaccinated and unvaccinated women and their infants were observed for safety outcomes with the exception of fever and chorioamnionitis. Compared to no vaccination, three studies showed a significantly increased relative risk for the presence of the ICD-9 code for chorioamnionitis in electronic patient data after pertussis vaccination. However, no study reported an increased risk for clinical sequelae of chorioamnionitis after vaccination during pregnancy, such as preterm birth or neonatal intensive care unit admission. Vaccine effectiveness against pertussis in infants of immunized mothers ranged from 69 to 91\% for pertussis prevention, from 91 to $94 \%$ for prevention of hospitalization and was 95\% for prevention of death due to pertussis. Risk of bias was serious to critical for safety outcomes and moderate to serious for effectiveness outcomes. GRADE evidence quality was moderate to very low, depending on outcome.

Conclusion: Although an increased risk for a diagnosis of fever and chorioamnionitis was detected in pregnant women after pertussis vaccination, there was no association with a higher frequency of clinically relevant sequelae. Vaccine effectiveness for prevention of infant pertussis, hospitalization and death is high. Pertussis vaccination during pregnancy has an overall positive benefit-risk ratio. In view of the overall quality of available evidence ongoing surveillance of chorioamnionitis and its potential sequelae is recommended when pertussis vaccination in pregnancy is implemented.

Trial registration: PROSPERO CRD42018087814, CRD42018090357.

Keywords: Tdap, Acellular pertussis vaccine, Pertussis, Pregnancy, Chorioamnionitis

\footnotetext{
* Correspondence: vygen-bonnets@rki.de

'Immunization Unit, Robert Koch Institute, Seestrasse 10, 13353 Berlin,

Germany

Full list of author information is available at the end of the article
}

(c) The Author(s). 2020 Open Access This article is distributed under the terms of the Creative Commons Attribution 4.0 International License (http://creativecommons.org/licenses/by/4.0/), which permits unrestricted use, distribution, and reproduction in any medium, provided you give appropriate credit to the original author(s) and the source, provide a link to the Creative Commons license, and indicate if changes were made. The Creative Commons Public Domain Dedication waiver (http://creativecommons.org/publicdomain/zero/1.0/) applies to the data made available in this article, unless otherwise stated. 


\section{Background}

Pertussis is a vaccine-preventable bacterial respiratory infection leading to high morbidity, especially in young infants. Disease burden of pertussis remains significant despite high vaccination coverage in children in western countries [1]. In Germany, annual pertussis incidence ranged from 11 to 20 per 100 , 000 inhabitants during the years 2013-2018 [2]. Young infants $<6$ months of age are at increased risk of pertussis related complications, such as otitis media, pneumonia, apnea, encephalopathy, as well as pulmonary hypertension which is caused by extreme lymphocytosis [3]. Severe and potentially lethal complications are most common in infants $<2$ months of age [4]. In Germany, mean annual incidence of pertussis among infants aged $\leq 3$ months was 80 per 100,000 during the past 5 years, while hospitalization rate in those young infants was $>75 \%$ [RKI, surveillance data, unpublished]. A recent German capturerecapture study suggests that incidences based on statutory surveillance are substantially underestimated by 39\% [5]. Since the introduction of nationwide mandatory pertussis reporting in Germany in 2013, two pertussis-related deaths were notified in infants 2 and 4 months of age [6] [RKI, surveillance data, unpublished]. Due to young age, this vulnerable group cannot benefit from direct effects of vaccination. Studies have shown that the majority of pregnant women in western countries have insufficient concentrations of pertussis-specific antibodies to confer protection to the newborn via diaplacentally transferred maternal antibodies [7-10]. In contrast, vaccination during pregnancy results in high levels of antibodies in the mother and the newborn [11, 12]. Therefore, vaccination of pregnant women with an acellular pertussis vaccine has been introduced in a number of countries, including the United Kingdom, USA, Belgium, Switzerland, Spain and Australia [13-19].

So far, six systematic reviews have investigated the effectiveness and/or safety of pertussis vaccination during pregnancy $[4,20-24]$. Importantly, however, a number of new studies on this topic were published only recently, and not all of these reviews addressed the entire spectrum of clinically relevant outcomes comprising safety as well as effectiveness for mother and child. Furthermore, some of the earlier reviews did not use the most advanced methodological tools recommended to address risk of bias and evidence quality, both being of key importance for decisionmaking regarding the implementation of vaccine programs during pregnancy.

We therefore performed a systematic review assessing the evidence for safety and effectiveness of pertussis vaccination during pregnancy.

\section{Methods}

\section{Search strategy and selection criteria}

The protocols of this systematic review were published in the Prospective Register for Systematic Reviews (PROSPERO; registration no, CRD42018087814 (for safety), CRD42018090357 (for effectiveness)). The review was performed according to the guidelines in the Preferred Reporting Items for Systematic Reviews and Meta-analyses (PRISMA) statement [25].

To be eligible, a study had to match the following PICO (population, intervention, comparator, outcome) criteria:

$\mathrm{P}$ - pregnant women and their newborns.

I - vaccination with an acellular pertussis componentcontaining vaccine during pregnancy.

C - placebo or no vaccination or vaccination with other, not pertussis component-containing vaccines, e.g. tetanus, tetanus-diphtheria, or influenza vaccination (only for effectiveness outcomes).

O - efficacy/effectiveness: (1) laboratory-confirmed pertussis in infant $\leq 3$ months of age; (2) hospitalization due to (1); (3) death due to (1);

$\mathrm{O}$ - safety: (4) fever $\left(\geq 38^{\circ} \mathrm{C}\right)$ in pregnant woman; (5) pre-eclampsia/eclampsia; (6) chorioamnionitis; (7) preterm birth; (8) stillbirth; (9) low birth weight; (10) malformation; (11) neonatal intensive care unit (NICU) admission; (12) neonatal sepsis; (13) neonatal death.

Electronic databases searched were MEDLINE and EMBASE (date of initial search: 26 February 2018; last update: 10 January 2019). For details on the complete search strategy, see Additional file 1: Figure S1. Additionally, the Cochrane Data Base of Clinical Trials was searched, and a search in ClinicalTrials.gov was conducted for unpublished or ongoing trials. Electronic searches were complemented by manually screening reference lists of all identified studies and those of identified reviews. Search results (titles, abstracts, full texts) were independently assessed by three investigators (WH, TH, SVB). Differences were discussed until a consensus was reached.

Search was limited to studies published from 01 January 2010 onwards. We did not make restrictions with regard to setting, language or publication status (published/unpublished).

\section{Data extraction}

Three independent reviewers (WH, TH, SVB) used standardized forms to extract study characteristics from eligible studies and to assess risk of bias. In case of disagreement, a final decision was made by consensus. The following data were extracted: study location, setting, study design, study period, participants, intervention, comparator, study size, outcomes, study sponsorship, conflict of interests, number (proportion) of vaccinated 
participants with outcome, number (proportion) of control participants with outcome, unadjusted estimates, adjusted estimates, and confounders.

\section{Assessment of risk of bias and quality of evidence}

For randomized controlled trials (RCTs), the Cochrane risk of bias tool was used to assess the following domains: random sequence generation, allocation concealment, blinding of participants and personnel, blinding of outcome assessment, incomplete outcome data, selective reporting, and other bias [26]. RCTs were categorized as being at "high risk", "low risk" or "unclear risk" of bias. For non-randomized studies, the ROBINS-I tool was used, comprising the following domains: bias due to confounding, bias in selection of participants into the study, bias in classification of interventions, bias due to deviations from intended interventions, bias due to missing data, bias in measurement of outcomes, and bias in selection of the reported results [27]. Risk of bias was categorized as being "low risk", "moderate risk", "serious risk" or "critical risk".

The methodology of the GRADE (Grading of Recommendations Assessment, Development and Evaluation) working group and the software "GRADE profiler" were used to assess the quality of evidence [28, 29].

\section{Statistical analysis}

Abstracted data were aggregated in tables. Risk ratios, odds ratios, risk differences and corresponding 95\% confidence intervals $(95 \% \mathrm{CI})$ were either calculated or extracted from the publications. A $p$-value $<.05$ was considered statistically significant. Vaccine effectiveness (VE) was either extracted from the publications or calculated as [1-(risk ratio or rate ratio comparing vaccine and control recipients) $] \times 100$. Since heterogeneity between studies was judged to be high with regard to setting, study design, outcome definition and confounders considered in the analysis, no metaanalyses were performed.

\section{Results}

\section{Search results}

By systematic literature search, a total of 1273 publications were identified. Screening of titles and abstracts led to the exclusion of 1074 publications. Of the remaining 199 studies, 22 were found to match our inclusion criteria (see flowchart and list of excluded studies in the Additional file 1: Figure S1 and Table S1). The characteristics of the included studies are listed in Table 1.

\section{Vaccine safety}

\section{Evidence base and risk of bias}

Three RCTs [30-32] and 11 non-randomized studies [15, 33-38, 40-42, 50] from Belgium, United Kingdom,
Canada, New Zealand, Vietnam and the USA reported maternal and/or infant safety outcomes (Table 2). Taking into account overlapping study populations of four studies based on the US Vaccine Safety Datalink project $[34,35,38,50]$, data from a total of 1.4 million pregnant women were included, of which 199,846 had received a pertussis-component-containing vaccine during pregnancy. In three RCTs [30-32] and one non-randomized study [42] the pertussis-containing vaccine used was Adacel ${ }^{\circ}$, whereas in four other studies it was Boostrix ${ }^{\circ}[15,37,52,53]$ and in the British study [36] it was Repevax ${ }^{\circ}$. In most studies from the US $[33-35,38,40,41,50]$ the vaccine used was not specified.

In most studies, women who had received tetanusdiphtheria-acellular pertussis (Tdap) vaccines (Adacel $^{\circ}$ or Boostrix $^{\circ}$ ) or Tdap-IPV-vaccines (Repevax ${ }^{\circ}$ ), were compared to women, who were either unvaccinated or had received placebo. In two RCTs, the comparison group was vaccinated with a tetanus-toxoid-containing vaccine [31, 32].

Risk of bias (RoB) was judged high for one [31] and low for two RCTs [30, 32] (Additional file 1: Table S2). Vaccine administrators were not blinded in the trials by Halperin et al. [32] and Munoz et al. [30], but we considered this to be unlikely to have influenced the outcomes "prematurity", "pre-eclampsia/eclampsia", "fever" and "malformations" in these studies.

Of the 11 non-randomized studies, we judged eight as having a serious RoB and three studies to show a critical RoB (Additional file 1: Table S2). The main reasons for these classifications were confounding, selection bias, and imprecise outcome assessment. Residual confounding could not be excluded in any of the studies. In addition, a likely healthy vaccinee bias was observed in most studies. Preexisting comorbidities (e.g. arterial hypertension [34, 38, 41], heart disease [38], diabetes [34, 41], pulmonary disease [34, 38]) and referral to high-risk obstetrics clinics [41] were more frequent in non-vaccinated women than in vaccinated women. In addition, health care utilization differed between vaccinated and non-vaccinated women (e.g. higher uptake of influenza vaccination [33, 37, 40,54] and ultrasound examinations $[40,54]$ during pregnancy among Tdap vaccinated women). In several studies, Tdap-vaccinated women showed indications for better uptake or earlier start of prenatal care [33, 38, 40, 41]. Frequently, these results were statistically significant $[33,38,41]$. Healthy vaccinee bias might have shifted estimates towards more favorable outcomes in vaccinated women and their infants. Moreover, with respect to preterm birth, immortal time bias could also have influenced the results.

In two studies [38, 39], a large proportion (74 and 79\% respectively) of eligible study participants was excluded from analysis, e.g., women with irregular health insurance 

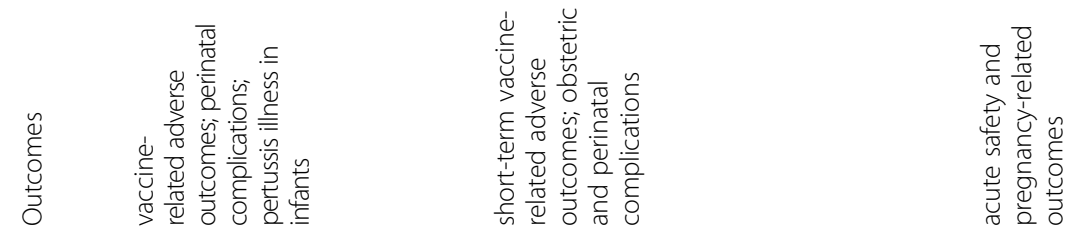

$z$ 宁 윰

$\stackrel{\infty}{+}$

$\stackrel{\infty}{n}$

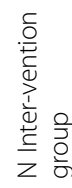

$m$

ธก

$\stackrel{+}{n}$

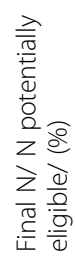

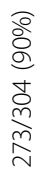

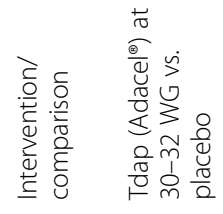

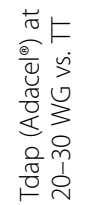

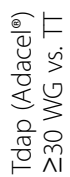

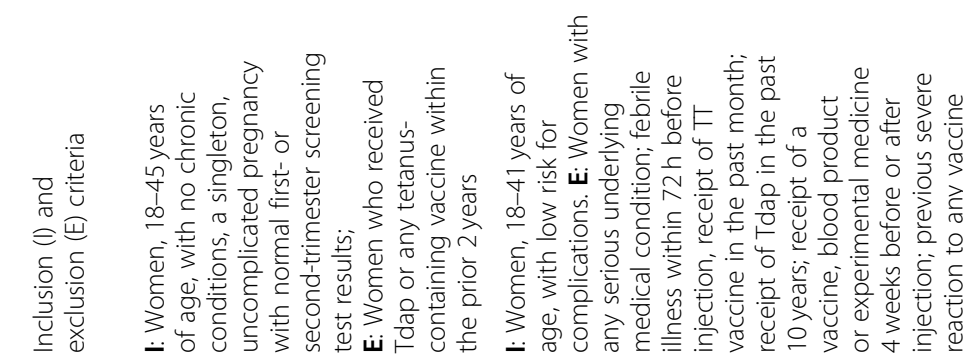

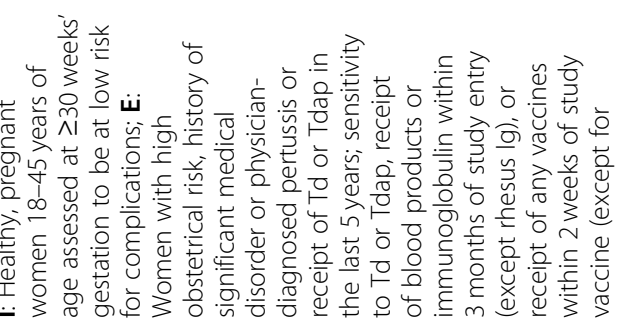

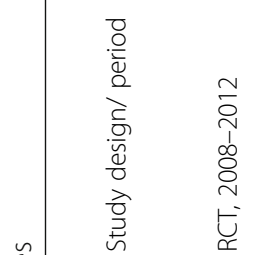

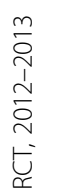

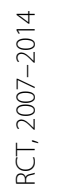

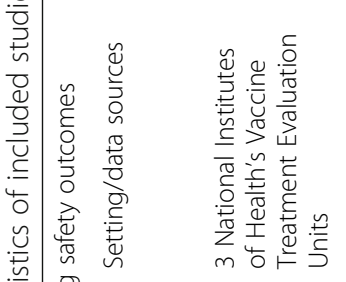

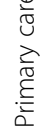

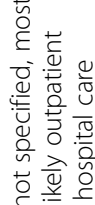

항

๖े

กั่

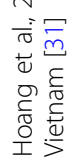

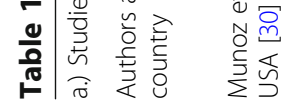

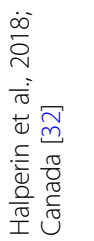




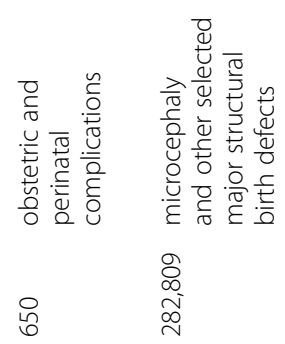

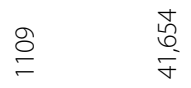

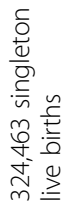

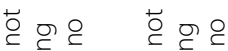

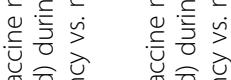

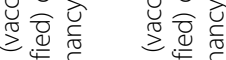

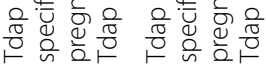
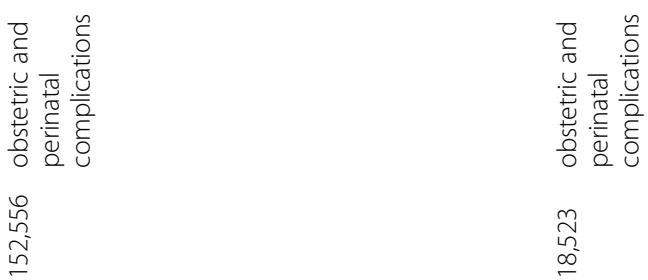

\begin{tabular}{l}
$m$ \\
\multirow{2}{*}{} \\
$\infty$
\end{tabular}

$\infty$
8
$\vdots$
$\dot{y}$
$\dot{y}$

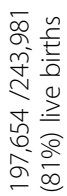

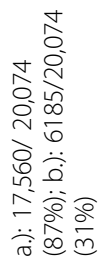

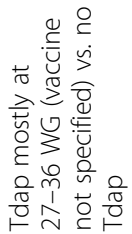

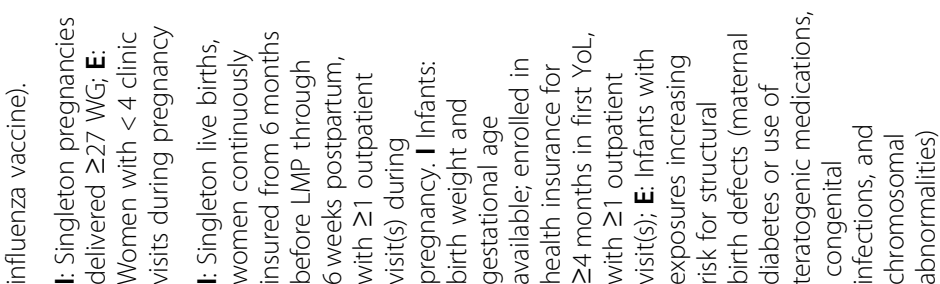

$m$
$\vdots$
1
0
0
0
$y$
0

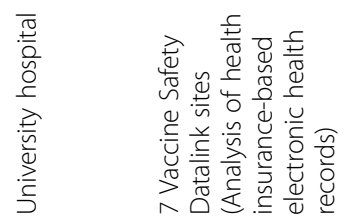

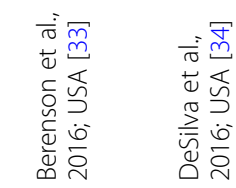

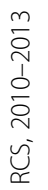

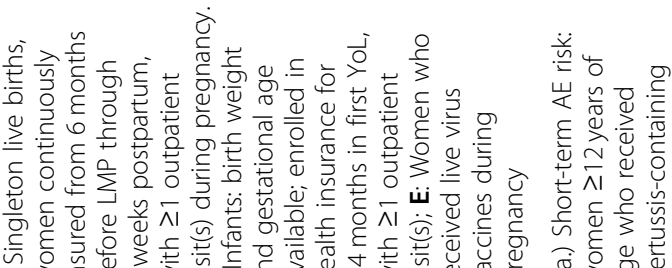

음

mำ

i 1

ì

牙宮
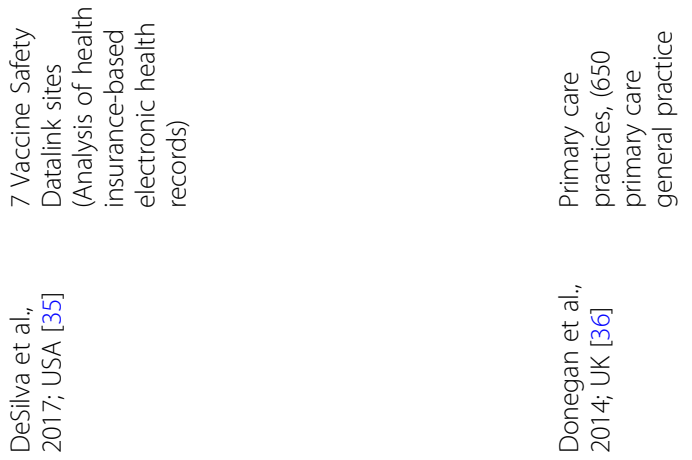

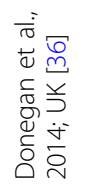




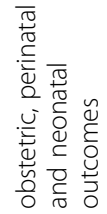

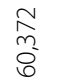

$\frac{\infty}{\infty}$

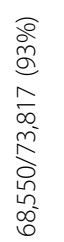

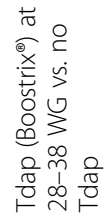

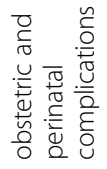

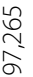

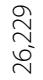

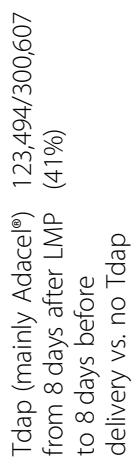

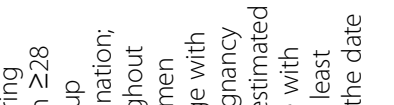

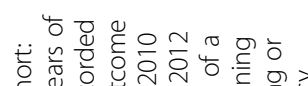

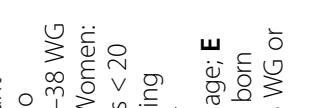

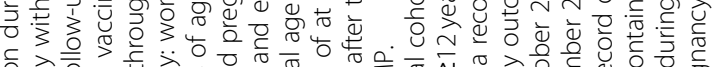
ᄃ

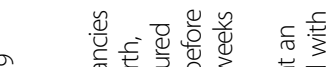

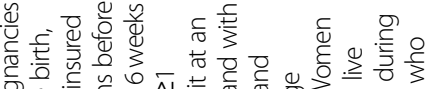

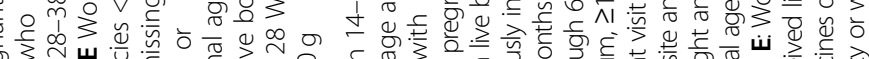

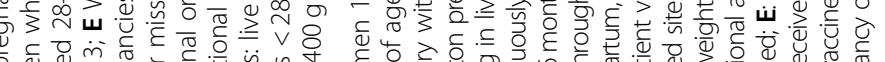

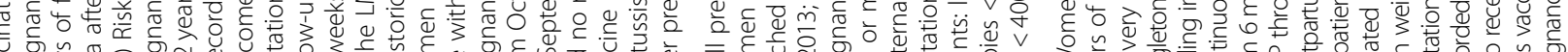

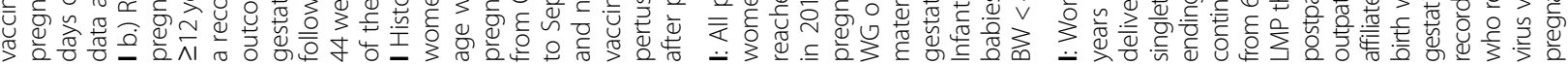

$\stackrel{m}{\grave{2}}$

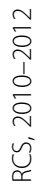

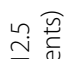

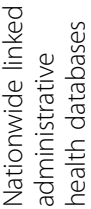

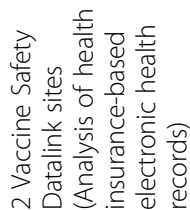

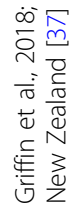

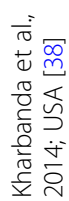




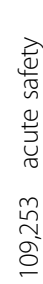
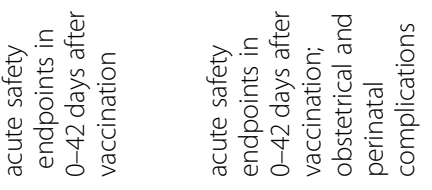

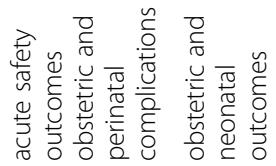

总

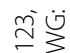

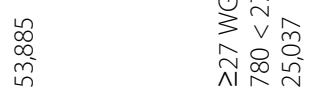

نㅊ

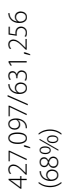

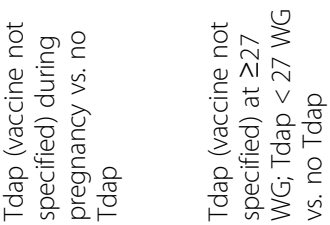

ฯ

ถิ

$\stackrel{N}{\frac{N}{\pi}}$

$\stackrel{\Upsilon}{z}$

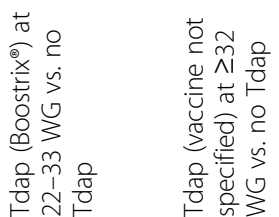

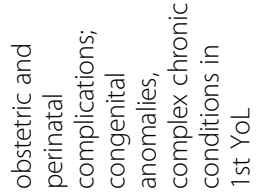

กิก

$\stackrel{\infty}{m}$

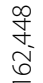

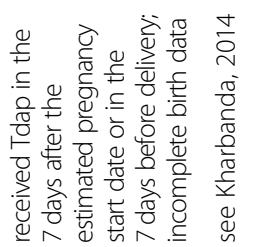

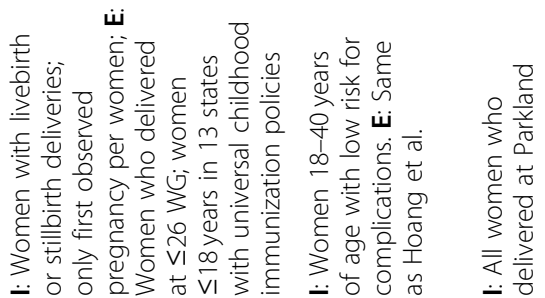

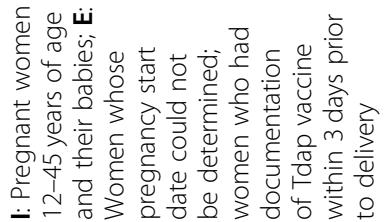

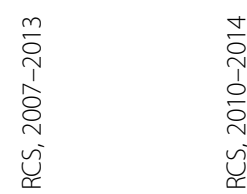

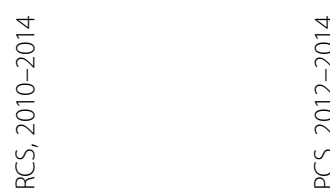

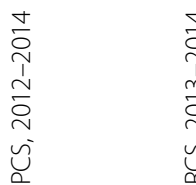

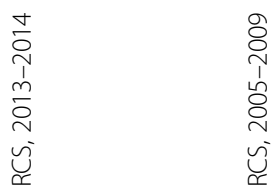
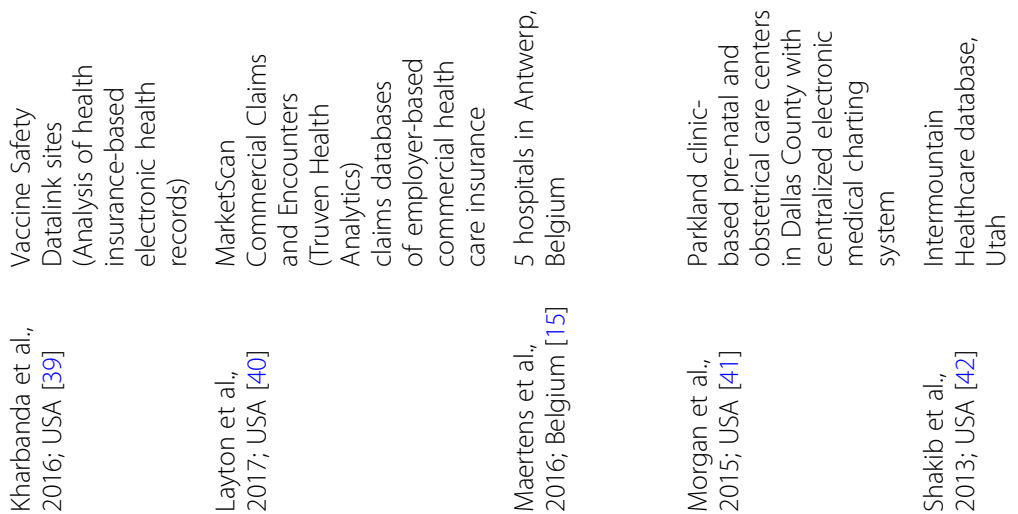

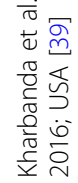

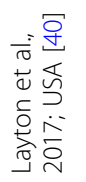

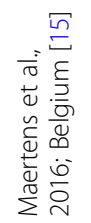

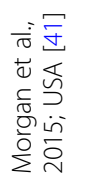

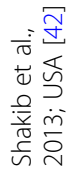




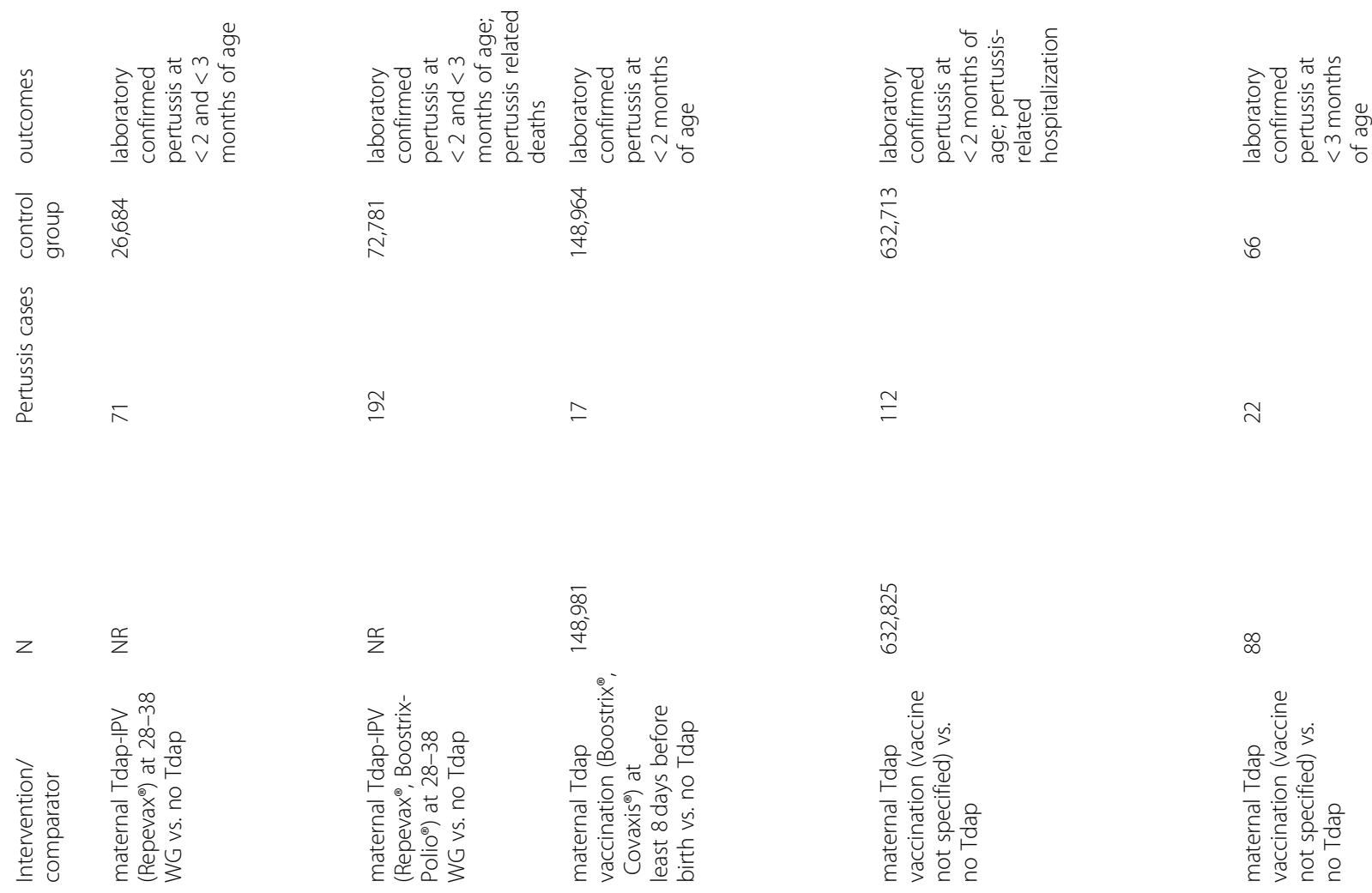
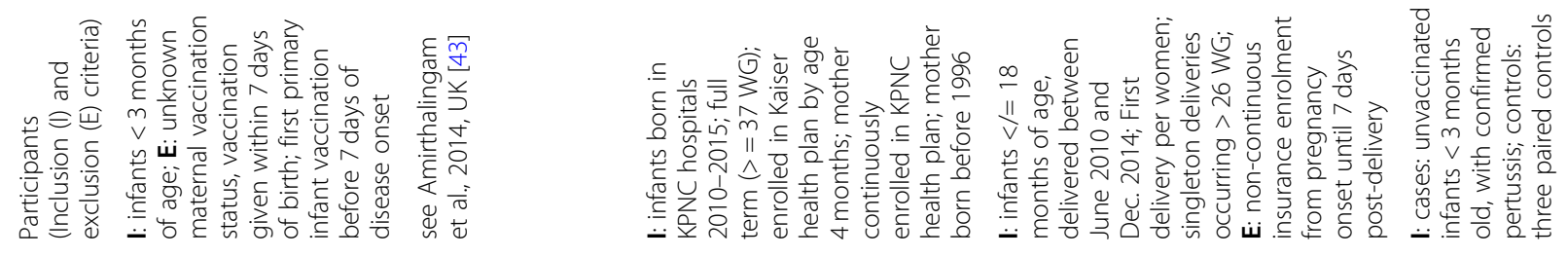

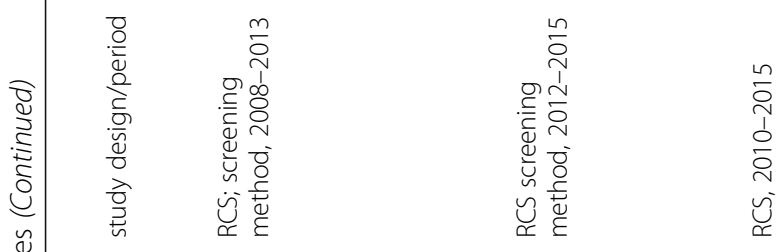
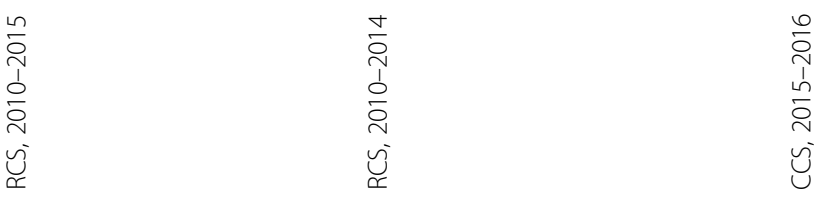

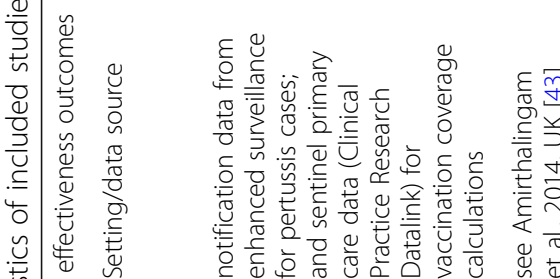
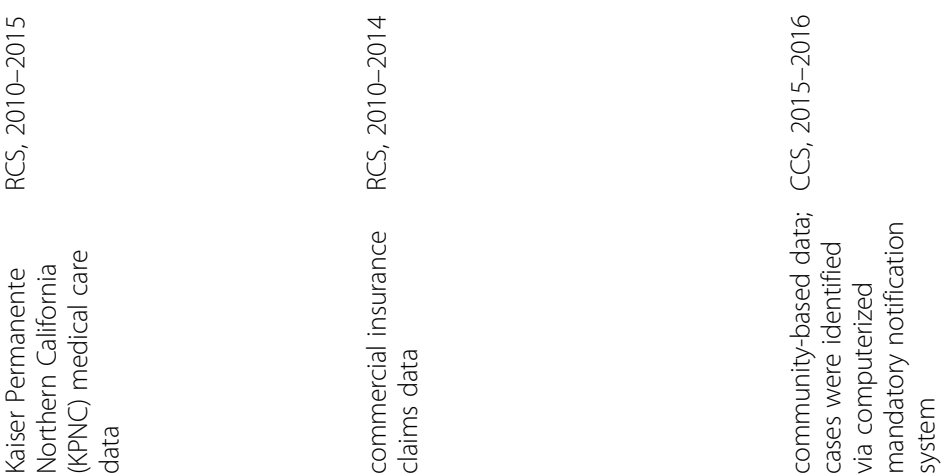

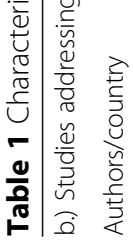

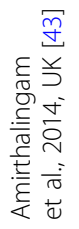

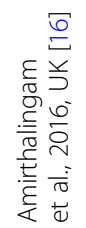

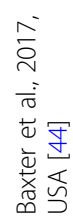

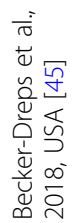

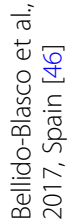




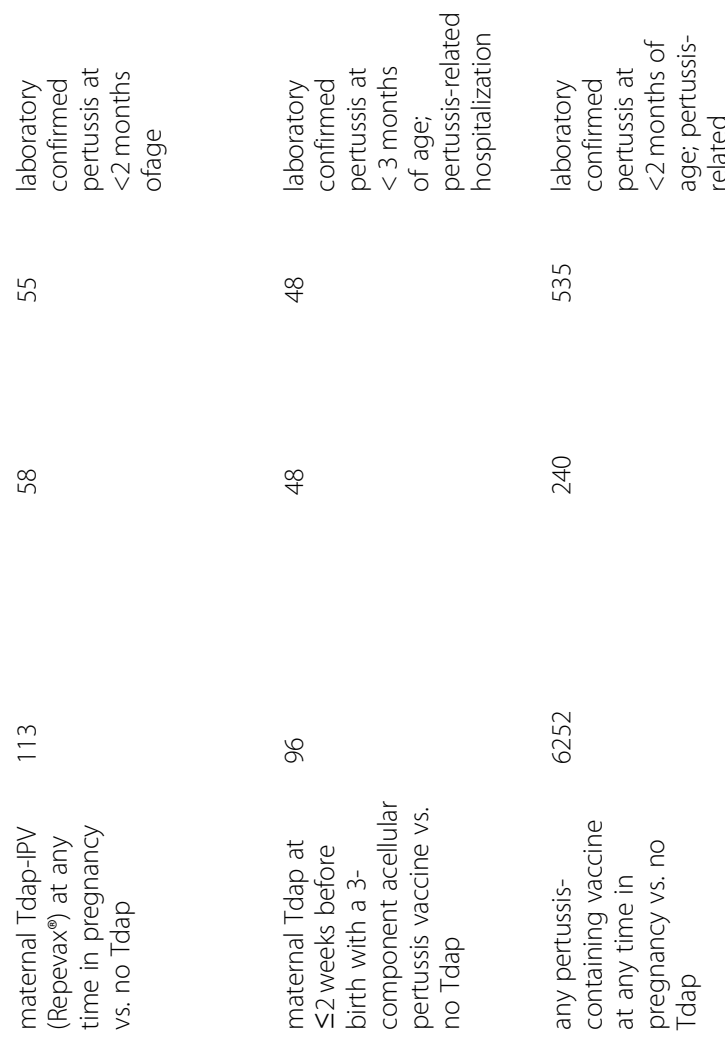

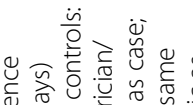

A1

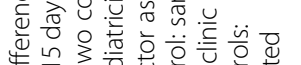

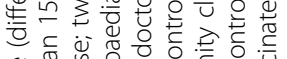

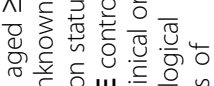

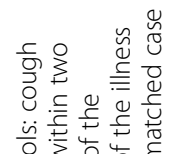

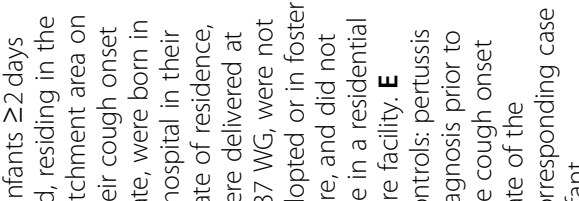

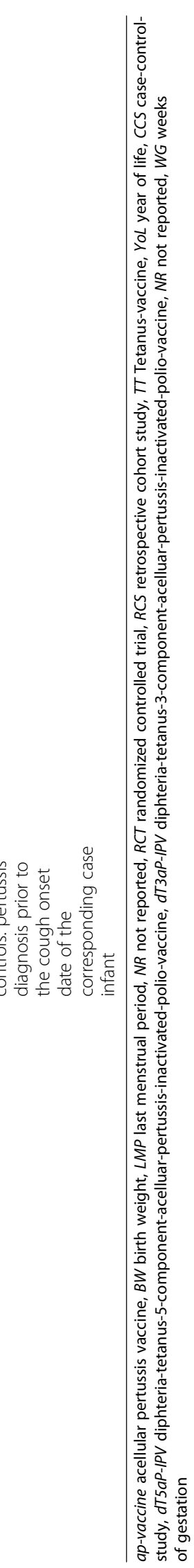

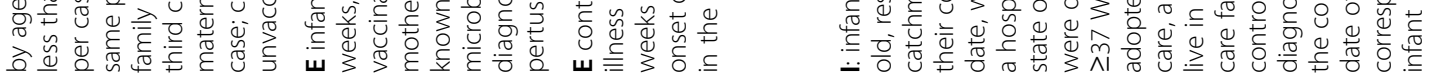
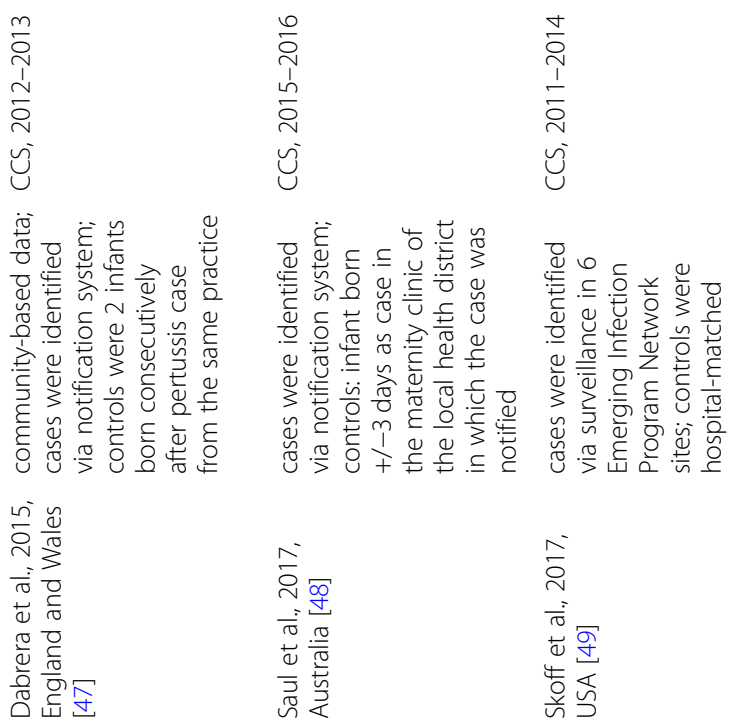


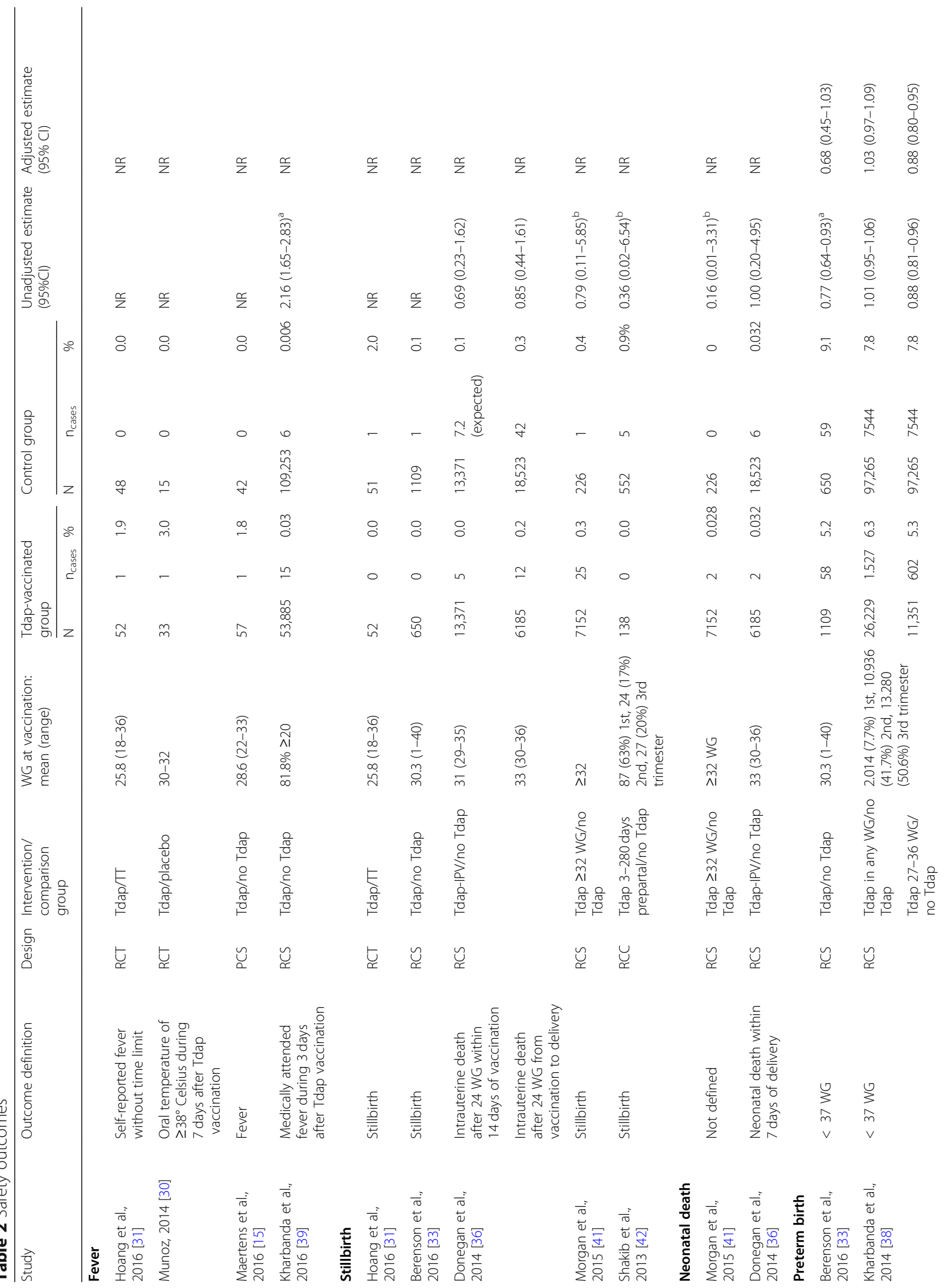




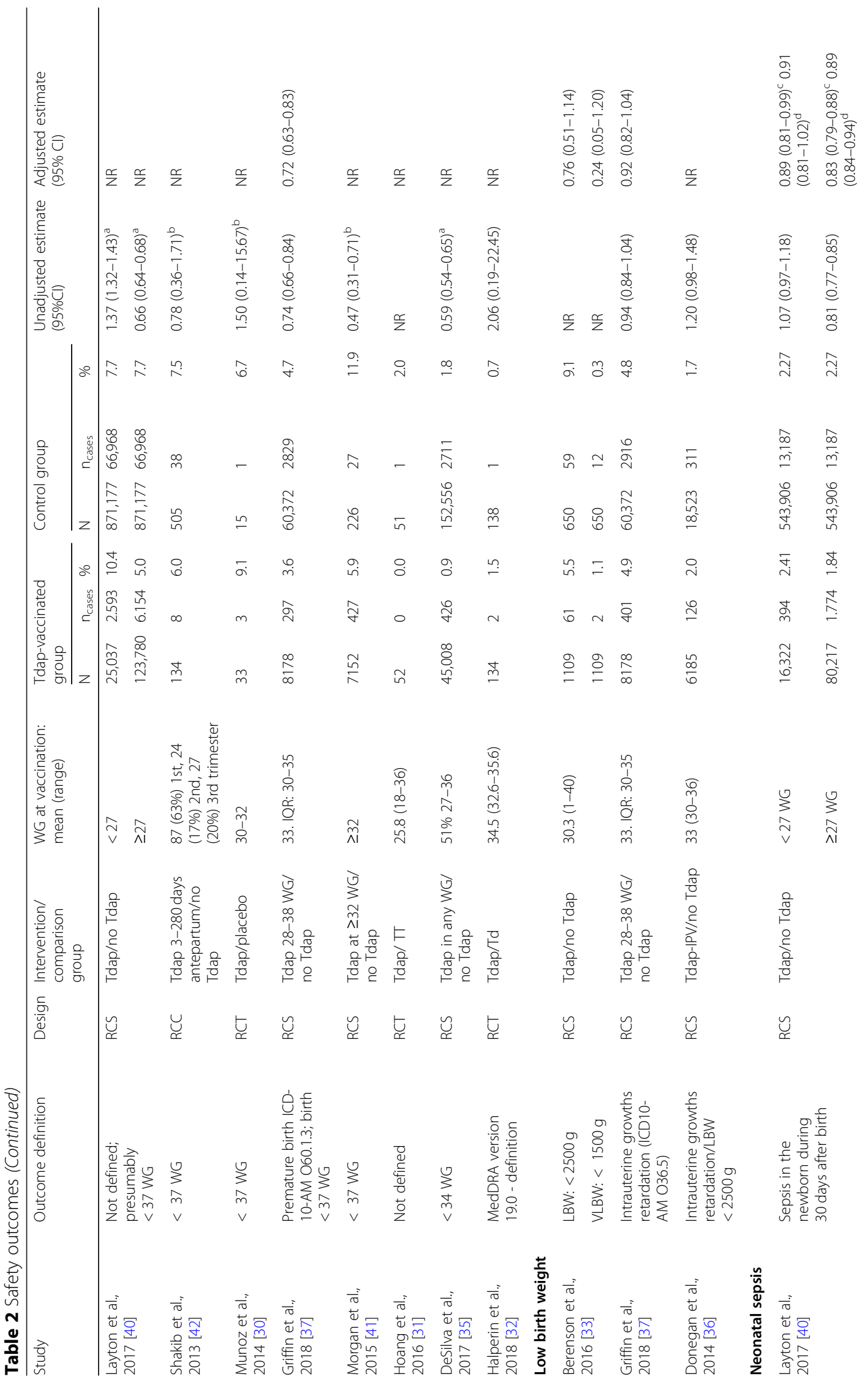




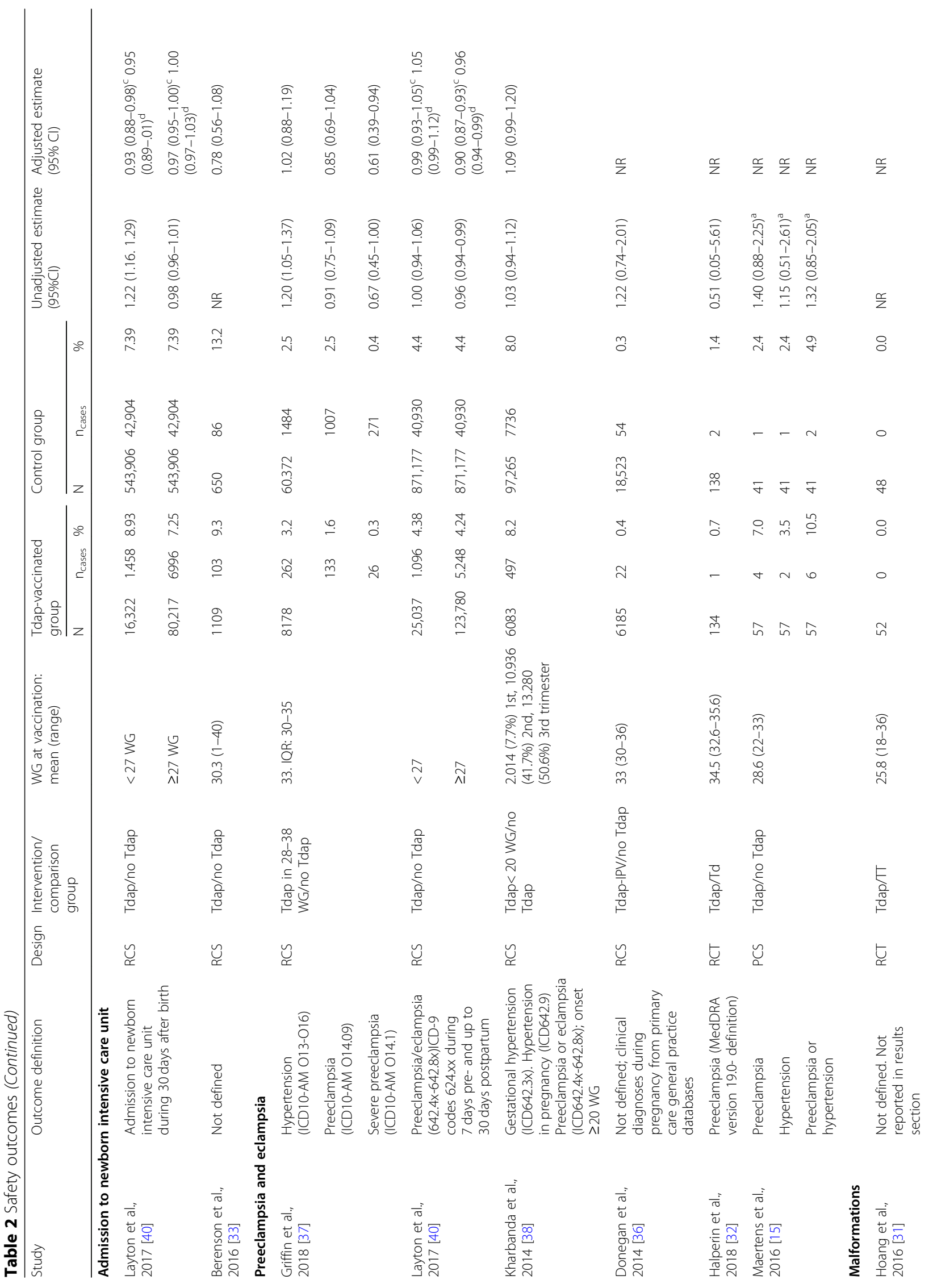




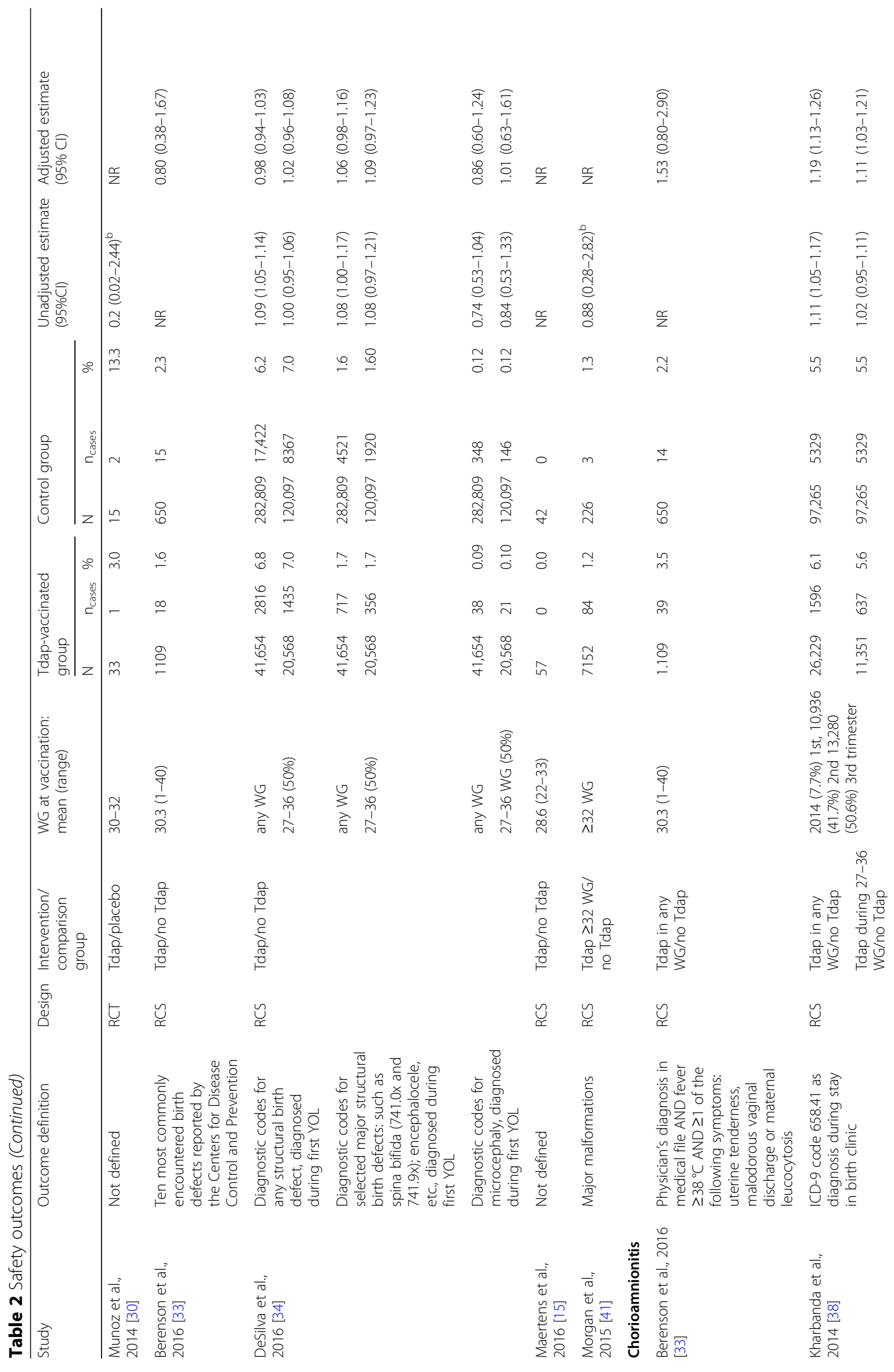




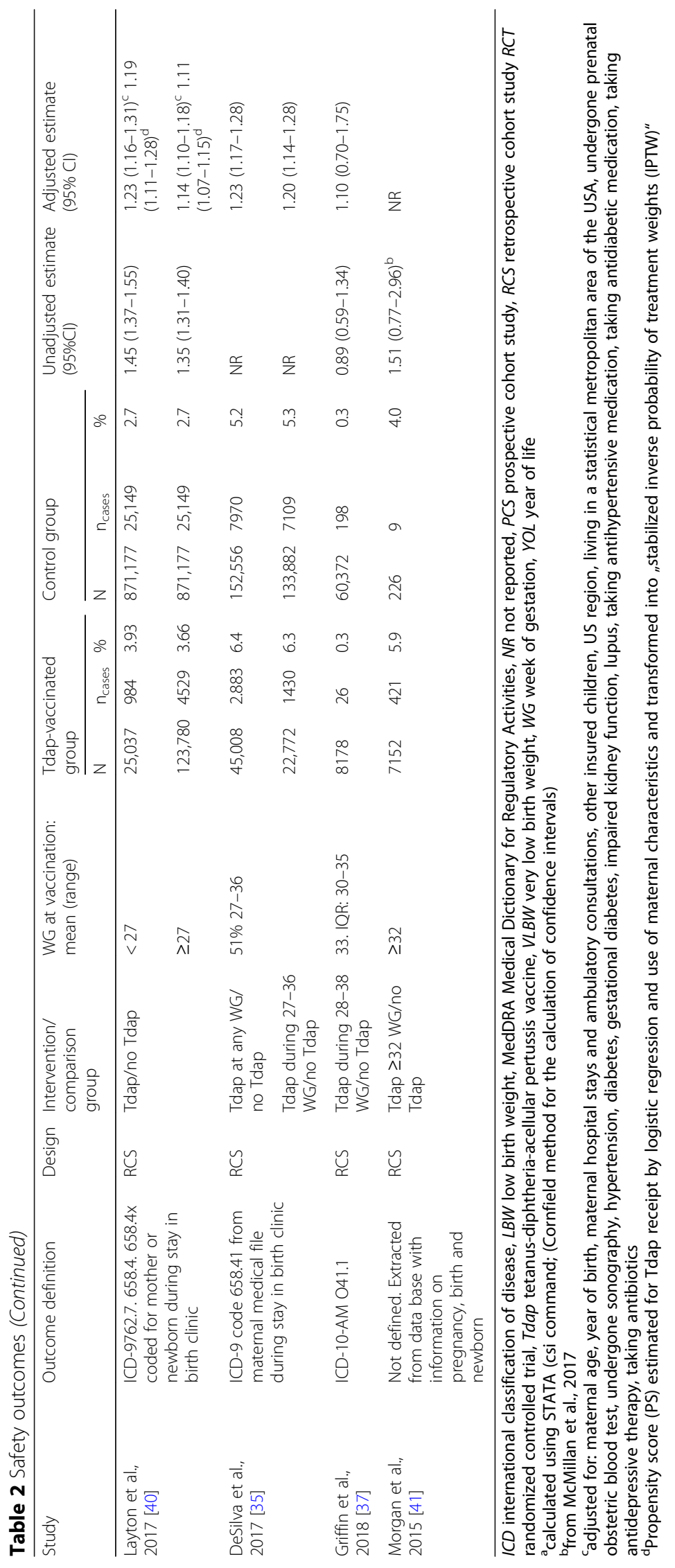


status or with a history of multiple pregnancies, stillbirth or premature birth. This might have limited generalizability of study results. In addition, exclusion of pregnancies ending in stillbirth or abortion might have resulted in selection bias with regard to potentially associated outcomes, like congenital malformations.

Five studies were based on commercial health data bases using ICD codes [34, 35, 38-40]. Coding for commercial reasons, such as insurance claims, might be prone to favoring more severe diagnoses. This might be a relevant source of bias for some outcomes, such as chorioamnionitis, but not for others, like admission to NICU. In studies, which were based on medical records $[33,36]$, no standardized case definitions were used, such as those developed by the Brighton Collaboration [55]. Here, potential misclassification was likely to be non-differential.

\section{Fever}

Rates of fever after Tdap vaccination in pregnancy were assessed in four studies [15, 30,31, 50]. The definition of fever varied considerably across studies (Table 2). Overall, fever following immunization was reported in 0.03 to $3 \%$ of pregnant women and occurred more frequently in Tdap-vaccinated women than in control women.

\section{Stillbirth}

One RCT [31] and four non-randomized studies [33, 36, $41,42]$ assessed stillbirths. None of these studies reported an increased risk in Tdap-vaccinated women.

\section{Neonatal deaths}

Two non-randomized studies reported on neonatal death $[36,41]$. A few cases were observed but there was no significant association with Tdap vaccination during pregnancy.

\section{Preterm birth}

Ten of the included studies reported on preterm birth, mostly defined as gestational age $<37$ weeks. In the three RCTs [30-32], only a few preterm births were observed without differences between vaccinated and control mothers. In seven non-randomized studies [33, 35, 37, $38,40-42]$, risk of preterm birth was higher in unvaccinated than in Tdap-vaccinated women. In one of these studies [40], compared to no vaccination, Tdapvaccination at $27-36$ weeks gestation was associated with a decreased risk of preterm birth, whereas earlier vaccination (before 27 weeks) was associated with an increased risk (see Table 2).

\section{Low birth weight}

Low birth weight (LBW; $<2500 \mathrm{~g}$ ) or very low birth weight (VLBW; < $1500 \mathrm{~g}$ ) were assessed in three studies.
Donegan et al. [36] reported on intrauterine growth retardation/LBW, Berensen et al. [33] assessed LBW and VLBW separately. Neither study reported an association between Tdap-vaccination during pregnancy and low birth weight. Griffin et al. [37] assessed the outcome "fetal growth restriction" and also found no association with Tdap-vaccination during pregnancy.

\section{Congenital malformations}

Definition and recording of congenital malformations varied considerably across the seven studies which reported on this outcome. Hoang et al. [31] did not detect any malformations within 30 days after birth, while Munoz et al. [31] observed one case of pyelectasia in an infant of a Tdap-vaccinated mother and two cases of cardiac malformation in infants whose mothers had not been vaccinated. In five non-randomized studies $[15,33$, $34,41,42]$, the authors observed no association between Tdap-vaccination during pregnancy and malformations including infants of mothers who were vaccinated during the first trimester in the study by DeSilva et al. [34].

\section{Neonatal septicaemia}

Only one non-randomized study [40] reported on neonatal sepsis, but did not distinguish between early- and late-onset sepsis. Newborns of Tdap-vaccinated mothers were at lower risk of septicemia than newborns of unvaccinated mothers.

\section{Admission to NICU}

Two non-randomized studies reported on NICU admission $[33,40]$. Risk of NICU admission was lower in newborns of vaccinated mothers than in those of unvaccinated mothers in both studies.

\section{Pre-eclampsia and eclampsia}

The six non-randomized studies that reported on preeclampsia and eclampsia used heterogeneous definitions for this outcome (see Table 2). Layton et al. [40] observed a slightly decreased risk for pre-eclampsia and eclampsia in women who had been vaccinated after 26 weeks of gestation, compared to unvaccinated women (RR: 0.96; CI: 0.94-0.99). Similar findings were obtained by Griffin et al. [37] for severe pre-eclampsia (RR: 0.61; CI: $0.39-0.94)$. In the remaining four studies $[15,32,36$, 38], no association between Tdap-vaccination and preeclampsia or eclampsia was observed.

\section{Chorioamnionitis}

Chorioamnionitis was investigated in six non-randomized studies [33, 35, 37, 38, 40, 41]. All of them reported an increased risk of chorioamnionitis in women who had received Tdap-vaccination during pregnancy (Table 2). Of those, two studies investigated Tdap given at any week of 
gestation, while in the remaining four studies Tdap vaccination was performed in the third trimester $(>=$ 27 to $>=32$ weeks of gestation). In the four largest studies comprising 8178 to 123,780 vaccinated women, the outcome was defined using ICD-codes $[35,37,38,40]$. One study [41] did not report the outcome definition. Only Berenson et al. [33] used a clinical case definition for identifying chorioamnionitis in electronic medical records. In the six studies, risk ratios ranged from 1.04 (95\%CI: $0.98-1.11)$ to 1.53 (95\%CI: $0.80-2.90)$. Size of risk estimates was unrelated to time point of Tdap vaccination during pregnancy. Estimates were statistically significant in three studies $[35,38,40]$.

In order to minimize the influence of health seeking behavior, Layton et al. [40] conducted a subgroup analysis restricting the cohort to pregnant women who were vaccinated against influenza. In this subgroup, the association between Tdap-vaccination and chorioamnionitis was weaker (adjusted RR: 1.09; 95\%CI: 1.03-1.15) than in the full cohort analysis (adjusted RR: 1.14; 95\%CI: 1.10-1.18). When propensity score adjustment was used, the estimate was no longer statistically significant.

\section{Vaccine effectiveness}

\section{Evidence base and risk of bias}

Eight studies fulfilled the inclusion criteria for the assessment of vaccine effectiveness (VE), including four cohort studies [16, 43-45] and four case-control-studies [46-49] (Table 2). The populations of the studies by Amirthalingam et al. [43] and Dabrera et al. [47] were included in the study by Amirthalingam et al. [16] (personal communication, Gavin Dabrera, October 17th, 2018). Taking into account this overlap, a total of 855 , 546 mother-infant-pairs were included in the studies, including 682 pertussis cases in infants $<3$ months (thereof $257<2$ months) of age and 854,864 non-cases. Mothers of 84 cases (12\%) and 205,919 non-cases (24\%) were vaccinated.

Five $[16,43,45,47,49]$ of the eight studies were judged as having a serious RoB, while three studies had a moderate RoB $[44,46,48]$. The main reasons for these classifications were selection bias, and imprecise outcome definitions. Using the screening method, Amirthalingam et al. [16, 43] could not adjust estimates for confounders other than age and time period. Residual confounding was judged likely to be present in the other studies as well, as adjustment for potential confounders was limited (see below). Therefore, and since there was evidence that vaccinated women had a more favorable health profile than those not vaccinated (e.g., uptake of influenza vaccination and ultrasound examination during pregnancy were more frequent in Tdap vaccinated women [45], smokers were more frequent in households of non-vaccinated women $[46,48])$, a "healthy vaccinee bias" appeared likely, suggesting that VE based on these studies might be overestimated.

The study by Skoff et al. [49] was judged as having a serious RoB because two-thirds of the initially identified study population was excluded and evidence for selection bias was found (the level of education and geographical distribution of study participants and excluded population differed significantly). The most common reasons for exclusion were non-reachability and missing consent to participate. We judged the most recent cohort study from the USA by Becker-Dreps et al. [45] as having a serious RoB because no clear case definition based on laboratory criteria was reported and the proportion of lab-confirmed cases in the subgroups was unknown.

\section{Pertussis in infants 0-3 months of age}

Three cohort $[16,43,44]$ and two case-control studies $[47,49]$ reported on the effectiveness of Tdap vaccination in pregnancy to prevent pertussis in infants $0-2$ months of age (Table 3). In all studies except for the one by Skoff et al. [49], only laboratory confirmed cases were included. Skoff et al. [49] also included cases with an epidemiological link or with a clinical picture of pertussis in their analysis (6\% of all cases). Confounderadjusted VE estimates in these five studies ranged from 78 to $93 \%$.

Four studies - the aforementioned two UK-based cohort studies by Amirthalingam et al. $[16,43]$ and two case-control studies from Spain and Australia [46, 48] reported vaccine effectiveness estimates for prevention of laboratory-confirmed pertussis in infants $0-3$ months of age between 69 und 91\% (Table 3). The age and timeperiod-adjusted point estimates of both studies by Amirthalingam et al. $[16,43]$ and the adjusted point estimate of the study by Bellido-Blasco et al. [46] were all 91\%. Bellido-Blasco et al. [46] adjusted their analysis for breastfeeding, maternal level of education and presence of other children in the household. The VE estimate of Saul et al. [48] of $69 \%$ (95\%CI: 13-89\%) was adjusted for breastfeeding, household size and gestational age.

\section{Pertussis-related hospitalization in infants $0-3$ months of age}

The confounder-adjusted VE estimates for the prevention of hospitalization due to pertussis in infants were 91 and $94 \%$ in two case-control studies from the US (California) [49] and Australia [48], respectively. Saul et al. [48] included infants $\leq 3$ months of age, whereas Skoff et al. [49] focused on infants $\leq 2$ months of age. The case definition used by Saul et al. [49] included only laboratory confirmed cases. Skoff et al. [49] included 6\% clinical cases without laboratory confirmation (Table 3). 


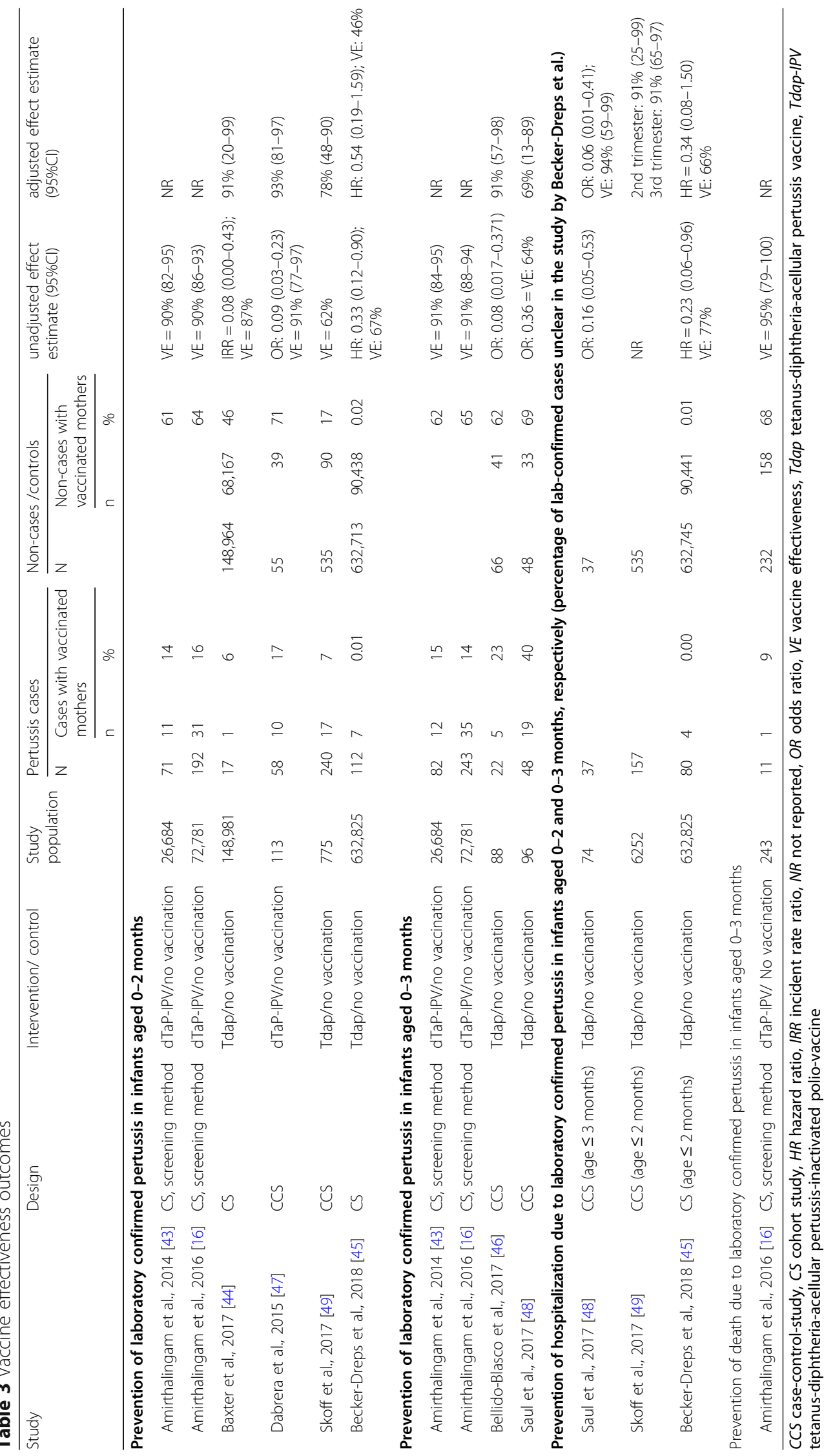


In the recent US American cohort study [45] which reported a VE of $66 \%$ for the prevention of hospitalization due to pertussis in infants $\leq 2$ months of age, the percentage of laboratory confirmed cases was unclear.

\section{Pertussis-related deaths in infants aged 0-3 months}

One cohort study from the United Kingdom [16] reported an effectiveness of $95 \%$ for Tdap vaccination in pregnancy for the prevention of death due to laboratory confirmed pertussis in infants 0-3 months of age (Table 3). Due to the use of the screening method, only an unadjusted effect estimate could be reported, which was based on 11 cases.

\section{Quality of evidence}

Regarding safety outcomes, quality of evidence according to GRADE was judged as low to very low. Reasons for downgrading were serious to critical risk of bias and imprecision. The evidence related to three of four effectiveness outcomes (pertussis $<2$ months of age, pertussis $<3$ months of age, pertussis-related death) was also downgraded to low quality due to moderate to serious risk of bias and inconsistency. Evidence quality for the remaining effectiveness outcome (hospitalization) was assessed as moderate due to risk of bias (see the GRADE evidence profile in Additional file 1: Table S3 for details).

\section{Discussion}

In this comprehensive systematic review, we evaluated the safety and effectiveness of acellular pertussis vaccination during pregnancy. Using data from more than 1.4 million pregnancies, we found similar risks for all prespecified safety outcomes in vaccinated and unvaccinated women and their infants except for two: slightly increased relative risks were detected for post-vaccination fever and chorioamnionitis at the time of delivery after Tdap vaccination in all studies reporting these outcomes. The risk increase was significant in one study reporting on maternal fever [50] and in three studies reporting on chorioamnionitis [35, 38, 40]. High effectiveness of Tdap-vaccination during pregnancy in preventing pertussis and related complications in the newborn and young infant was observed in all studies. Using GRADE methodology, the overall quality of evidence was rated as moderate to very low, depending on the outcome category.

Fever is a well-known adverse event after Tdap vaccination occurring at similar $[30,40,56]$ or lower $[40,57]$ frequency in pregnant compared to non-pregnant women. The variation in the reported rates in the four included studies can be explained by the differences in the outcome assessments and fever definitions (self-reported versus measured versus medically attended fever). Based on the largest cohort study's estimate [50], we calculated that about 6 additional cases of fever per 100,
000 pregnant women would occur after Tdap vaccination, as compared to no vaccination (using the difference of absolute risks in vaccinated versus nonvaccinated women).

Six studies reported a small increased relative risk of chorioamnionitis after Tdap vaccination. Three studies showed a significantly increased risk. All three were performed in the USA and had very large numbers of participants. They used "presence of respective ICD-9 codes in electronic patient data" as definition of chorioamnionitis $[35,38,40]$.

Chorioamnionitis is an inflammation of the fetal membranes, the amniotic cavity including its fluid and of the placenta, predominantly due to ascending bacterial infections $[58,59]$. It may occur at any time during pregnancy or delivery and may be preceded [59] or followed [58] by premature rupture of membranes. Chorioamnionitis is defined by either clinical features [58, 60], microbiological findings, histopathological signs [59] or a combination of these. Clinically relevant chorioamnionitis is a frequent cause of preterm birth and may lead to neonatal sepsis [59]. In the USA, a clinical and/or histologically proven chorioamnionitis is diagnosed in 40 $70 \%$ of preterm deliveries and in $1-13 \%$ of term deliveries [59]. From an immunological perspective, it appears plausible that vaccination can trigger an inflammatory process in pregnancy. During the course of pregnancy, the immune system of the expectant mother undergoes changes in its actiity, ranging from local inflammation that accompanies the tumor-like implantation of the fetus (first trimester) to the predominance of immune tolerance (second trimester) and ending up with inflammatory signals that lead to the induction of labor (third trimester) [61]. Tdap vaccination is only one of multiple activating stimuli to the maternal immune system during pregnancy. To our knowledge, so far no studies on pregnant animals have been published that examined the consequences of immune stimulation by vaccines for the outcome of pregnancy.

If chorioamnionitis were causally related to Tdapvaccination in the studies that were included in this review, for instance through some as yet unknown immunologic mechanism, we would expect increased risks of preterm birth or sepsis in infants of Tdap-vaccinated women. However, in seven studies [33, 35, 37, 38, 40-42], including those reporting a significant association between Tdap vaccination and chorioamnionitis, rates of preterm birth were even lower in Tdap-vaccinated than in unvaccinated women. Furthermore, in three studies the risk of NICU admission [33, 40] and sepsis [18] was also lower in infants of Tdap-vaccinated mothers as compared to infants of unvaccinated mothers, including one of the studies that reported an increased risk of chorioamnionitis [33, 40]. 
Hypothesizing that ICD-codes derived from electronic databases might not correctly reflect the clinical, microbiologic or histopathologic diagnosis of chorioamnionitis, Kharbanda et al. [38] validated the diagnosis by subgroup analysis. They randomly selected a validation sample of 220 women with hospital discharge ICD-codes for chorioamnionitis from electronic health charts. "Probable chorioamnionitis" was defined as the presence of ICD-9-Code 658.41 in combination with at least two clinical signs (maternal or fetal tachycardia, uterine tenderness, purulent or foul smelling amniotic fluid). Based on this definition the authors calculated that the positive predictive value (PPV) of the ICD-code for "probable chorioamnionitis" was $50 \%$. When applying this PPV to the whole study population the association between Tdap vaccination in pregnancy and chorioamnionitis remained statistically significant. However, for the subgroup of women vaccinated between 27 and 36 weeks gestation, the association was no longer statistically significant $(p=0.07)$.

Based on our analyses healthy vaccinee bias is a likely confounding factor in most studies, irrespective of study design. This could be an explanation for lower risks for potential sequelae of chorioamnionitis and for the increased frequency of diagnosing and coding chorioamnionitis as a consequence of better ante- and perinatal care including a more careful surveillance of vaccinated women (detection bias).

One possible explanation for the association between Tdap vaccination in pregnancy and chorioamnionitis might be confounding with epidural anesthesia. In a secondary analysis of data from a randomized trial, Abramovici et al. [62] reported a statistical association between use of epidural anesthesia and chorioamnionitis defined as the presence of fever and a physician's diagnosis warranting antibiotics. In this study, placental histopathologic examination revealed acute inflammation in $70 \%$ of those cases of clinical chorioamnionitis in which placental pathology was available for review (64\%). Placental culture was not performed. In the cohort study by Maertens et al. [15], 70\% of vaccinated versus $57 \%$ of unvaccinated women received epidural anesthesia, and in the subgroup analysis of Kharbanda et al. $95 \%$ of the 220 women with ICD-9 codes for chorioamnionitis had received an epidural anesthesia and 91\% antibiotic treatment (all 220 women had received Tdap vaccination) [38]. However, this information was not available for the whole study population. Epidural anesthesia is often associated with prolonged labor and maternal fever [63, 64], often leading to prophylactic antibiotic use [64]. Transient or non-specific maternal fever might thus get coded as chorioamnionitis [62], leading to an overcoding of this diagnosis in women who had received epidural anesthesia.
As vaccinated women in the 3 studies with a significant association between Tdap-vaccination and chorioamnionitis obtained better prenatal care (earlier and more frequent ante-natal clinic visits [38] and more frequently ultrasound examinations [40]), they may also have requested epidural anesthesia more frequently than non-vaccinated women. Unfortunately, rates of epidural anesthesia were not analyzed in any of the studies investigating chorioamnionitis after Tdap vaccination.

In our systematic review, vaccine effectiveness data of 855,546 mother-infant-pairs from Australia, Spain, UK, and the USA were analyzed. In the USA, Tdap vaccination in pregnancy has been recommended since 2011 [65], in Australia and Valencia (Spain) since 2015 [46, 48]. In the UK, pertussis vaccination in pregnancy was introduced in 2012 as an emergency measure during a nationwide pertussis outbreak with 14 infant deaths [13]. In 2014, it was decided to continue with the program, since pertussis incidence remained high in the overall population and the available evidence showed good safety and effectiveness of the intervention [16, 66]. Compared to safety studies, VE studies in our review had a lower risk of bias and higher quality of evidence. In all studies considering laboratory confirmed pertussis as the outcome, VE was high: it ranged from 69 to $91 \%$ for prevention of pertussis, from 91 to $94 \%$ for prevention of hospitalization and was $95 \%$ for prevention of death in infants $0-3$ months of age. The effect was diluted by additional inclusion of clinically suspected cases without laboratory pertussis confirmation in the study by Becker-Dreps [45], resulting in lower vaccine effectiveness estimates.

Our up-to-date systematic review has several strengths. We focus entirely on clinical outcomes (rather than immunological [serological] markers) and include a critical evaluation of a recently detected possible safety signal, i.e. chorioamnionitis. Using ROBINS-I, we applied the most advanced $\mathrm{ROB}$ tool to assess internal validity of the included observational studies, allowing a very detailed judgement. However, our review also has limitations that are mainly due to the limitations of the included studies. The majority of studies investigating safety outcomes had a considerable risk of bias, which impairs the ability of drawing firm conclusions on the risk of adverse events. Moreover, the three RCTs were designed and powered for the assessment of the immune response in pregnancy and, thus, were hampered by participant numbers that were too small for the assessment of rare safety outcomes. Regarding studies that investigated VE outcomes, those with the highest numbers of participants used the screening method to calculate VE. Since this method uses population estimates rather than individual data, controlling for confounders was not possible. 


\section{Conclusions}

In this systematic review we summarize the currently available evidence on safety and effectiveness of pertussis vaccination in pregnancy. Vaccine effectiveness for prevention of infant pertussis, hospitalization and death is high. Two safety issues were observed in the included studies: fever and chorioamnionitis. Six additional cases of fever per 100,000 vaccinated women are to be expected, which is a small number and makes fever an adverse event of minor importance. Increased ICD-coding of chorioamnionitis, even though statistically associated with Tdap vaccination during pregnancy in some of the studies, does not seem to be clinically relevant. However, when implementing Tdap vaccination in pregnancy, surveillance of all safety endpoints, including chorioamnionitis and its sequelae, is needed in view of a likely residual healthy vaccinee bias in currently available studies and in view of the overall low quality of the evidence. Given the high vaccine effectiveness, pertussis vaccination during pregnancy has an overall positive benefitrisk ratio, particularly if the incidence of pertussis in infancy is high.

\section{Supplementary information}

Supplementary information accompanies this paper at https://doi.org/10. 1186/s12879-020-4824-3.

Additional file 1: Figure S1. Systematic review on safety and effectiveness of pertussis vaccination in pregnancy; flowchart selection of included studies. Table S1. Systematic review on safety and effectiveness of pertussis vaccination in pregnancy; excluded studies. Table S2. Systematic review on safety and effectiveness of pertussis vaccination in pregnancy; results of the risk of bias (ROB) assessment. Table S3.

Systematic review on safety and effectiveness of pertussis vaccination in pregnancy; GRADE Evidence profile.

\section{Abbreviations \\ 95\% Cl: 95\% confidence interval; GRADE: Grading of Recommendations Assessment, Development and Evaluation; ICD: International classification of diseases; LBW: Low birth weight; NICU: Neonatal intensive care unit; PICO: Population, intervention, comparator, outcome; PRISMA: Preferred Reporting Items for Systematic Reviews and Meta-analyses; RCT: Randomized controlled trial; RoB: Risk of bias; RR: Relative risk; Tdap: Tetanus-diphtheria- acellular pertussis; VE: Vaccine effectiveness; VLBW: Very low birth weight}

\section{Acknowledgments}

The authors would like to thank Dr. Eva Hummers, Göttingen/Germany, who is a member of the pertussis working group of the German Standing Committee on Vaccination (STIKO), for contributing to the discussions of our results.

\section{Authors' contributions}

All authors were involved in the conception and design of the study and interpretation of the results. WH, SVB and TH performed the search and were responsible for data extraction, data analysis and drafting of the article. EG, RVK, MRM, CB, and UH revised the manuscript critically and contributed to the final draft. All authors have read and approved the manuscript.

\section{Availability of data and materials}

The full dataset is available from the corresponding author upon request.

Ethics approval and consent to participate

Not applicable.

\section{Consent for publication}

Not applicable.

\section{Competing interests}

CB, EG, MRM, RvK, SVB, TH, WH declare that there is not conflict of interest related to the topic presented in this paper.

UH is a member of the "Global Pertussis Initiative" (supported by Sanofi Pasteur, USA) and the "Collaboration of European Experts on Pertussis Awareness Generation", CEEPAG (supported by Sanofi, France).

\section{Author details}

${ }^{1}$ Immunization Unit, Robert Koch Institute, Seestrasse 10, 13353 Berlin, Germany. ${ }^{2}$ Leibniz Institute for Prevention Research and Epidemiology - BIPS, Bremen, Germany. ${ }^{3}$ Institute of Social Pediatrics and Adolescent Medicine, Division of Pediatric Epidemiology, Ludwig-Maximilians-University, Munich, Germany. ${ }^{4}$ Institut für klinische Mikrobiologie, Immunologie und Hygiene, Universitätsklinikum Erlangen und Friedrich-Alexander-Universität (FAU) Erlangen-Nürnberg, Erlangen, Germany. ${ }^{5}$ Medical Immunology Campus Erlangen, FAU Erlangen-Nürnberg, Erlangen, Germany. ${ }^{6}$ University of Basel Children's Hospital, Infectious Diseases and Vaccinology, Basel, Switzerland.

${ }^{7}$ Working Group on Immunization of the federal state of Bavaria (LAGI), Munich, Germany. ${ }^{8}$ Private practice for gynecology/obstetrics, Munich, Germany.

Received: 23 October 2019 Accepted: 24 January 2020

Published online: 13 February 2020

\section{References}

1. Yeung KHT, Duclos P, Nelson EAS, Hutubessy RCW. An update of the global burden of pertussis in children younger than 5 years: a modelling study. Lancet Infect Dis. 2017;17(9):974-80.

2. Robert Koch Institut. Infektionsepidemiologisches Jahrbuch meldepflichtiger Krankheiten für 2018. Berlin: Robert Koch Institute; 2019.

3. Cherry JD. Pertussis in Young Infants Throughout the World. Clin Infect Dis. 2016;63(suppl_4):S119-22.

4. Baclic O, Tunis M, Rotondo J, Saboui M, Duchesne-Belanger S, Brophy J, Chevalier T, Moffatt C, for the National Advisory Committee on Immunization (NACl). Literature Review on Immunization in Pregnancy with Tetanus Toxoid, Reduced Diphtheria Toxoid and Reduced Acellular Pertussis (Tdap) Vaccine: Safety, Immunogenicity and Effectiveness: An Advisory Committee Review Advisory Committee on Immunization (NACl). Ottawa: National Advisory Committee on Immunization (NACl); 2018. http:// publications.gc.ca/collections/collection_2018/aspc-phac/HP40-208-2018eng.pdf

5. Schielke A, Takla A, von Kries R, Wichmann O, Hellenbrand W. Marked Underreporting of Pertussis Requiring Hospitalization in Infants as Estimated by Capture-Recapture Methodology, Germany, 2013-2015. Pediatr Infect Dis J. 2018:37(2):119-125(7).

6. Robert Koch Institut. Infektionsepidemiologisches Jahrbuch meldepflichtiger Krankheiten für 2016. Berlin; 2017.

7. Gonik B, Puder KS, Gonik N, Kruger M. Seroprevalence of Bordetella pertussis antibodies in mothers and their newborn infants. Infect Dis Obstet Gynecol. 2005;13(2):59-61.

8. Smallenburg LCS, van Welie NA, Elvers LH, van Huisseling JCM, Teunis PFM, Versteegh FGA. Decline of IgG pertussis toxin measured in umbilical cord blood, and neonatal and early infant serum. Eur J Clin Microbiol Infect Dis. 2014;33(9):1541-5.

9. Shakib JH, Ralston S, Raissy HH, Stoddard GJ, Edwards KM, Byington CL. Pertussis antibodies in postpartum women and their newborns. J Perinatol. 2010;30(2):93-7.

10. Healy CM, Munoz FM, Rench MA, Halasa N, Edwards KM, Baker CJ. Prevalence of pertussis antibodies in maternal delivery, cord, and infant serum. J Infect Dis. 2004;190(2):335-40. 
11. Gall SA, Myers J, Pichichero M. Maternal immunization with tetanusdiphtheria-pertussis vaccine: effect on maternal and neonatal serum antibody levels. Am J Obstet Gynecol. 2011;204(4):334.e331-5.

12. Hardy-Fairbanks AJMD, Pan SJBS, Decker MDMDMPH, Johnson DRMDMPH, Greenberg DPMD, Kirkland KBMD, Talbot EAMD, Bernstein HHDOM. Immune responses in infants whose mothers received Tdap vaccine during pregnancy. Pediatr Infect Dis J. 2013;32(11):1257-60.

13. Joint Committee on Vaccination and Immunisation. Minute of teleconference on Thursday Wednesday 30 August 2012 10.00am - 12. 00am and post-teleconference discussion [https://app.box.com/s/iddfb4 ppwkmtjusir2tc/file/229171684750].

14. Vizzotti C, Neyro S, Katz N, Juarez MV, Perez Carrega ME, Aquino A, Kaski Fullone F. Maternal immunization in Argentina: a storyline from the prospective of a middle income country. Vaccine. 2015;33(47):6413-9.

15. Maertens K, Caboré RN, Huygen K, Hens N, Van Damme P, Leuridan E. Pertussis vaccination during pregnancy in Belgium: Results of a prospective controlled cohort study. Vaccine. 2016:34(1):142-50.

16. Amirthalingam G, Campbell H, Ribeiro S, Fry NK, Ramsay M, Miller E, Andrews N. Sustained effectiveness of the maternal pertussis immunization program in England 3 years following introduction. Clin Infect Dis. 2016;63: S236-43.

17. Australien Gouvernment, Department of health. National Immunisation Program Schedule [https://beta.health.gov.au/health-topics/immunisation/ immunisation-throughout-life/national-immunisation-program-schedule].

18. Bont $\mathrm{L}$, Bekker $\mathrm{M}$, Rots $\mathrm{N}$. Infection prevention in newborns through maternal vaccination: current insights and developments. Ned Tijdschr Geneeskd. 2016;160:D411.

19. Grawe C. Impfen in der Schwangerschaft ein Paradigmenwechsel In: Impfforum 732019; 2019.

20. Furuta M, Sin J, Ng ESW, Wang K. Efficacy and safety of pertussis vaccination for pregnant women - a systematic review of randomised controlled trials and observational studies. BMC Pregnancy Childbirth. 2017;17(1):390.

21. McMillan M, Clarke M, Parrella A, Fell DB, Amirthalingam G, Marshall HS. Safety of tetanus, diphtheria, and pertussis vaccination during pregnancy: a systematic review. Obstet Gynecol. 2017;129(3):560-73.

22. Gkentzi D, Katsakiori P, Marangos M, Hsia Y, Amirthalingam G, Heath PT, Ladhani S. Maternal vaccination against pertussis: a systematic review of the recent literature. Arch Dis Child Fetal Neonatal Ed. 2017;102(5):456-63.

23. Abu Raya B, Edwards KM, Scheifele DW, Halperin SA. Pertussis and influenza immunisation during pregnancy: a landscape review. Lancet Infect Dis. 2017;17(7):e209-e222.

24. Campbell H, Gupta S, Dolan GP, Kapadia SJ, Kumar Singh A, Andrews N, Amirthalingam $\mathrm{G}$. Review of vaccination in pregnancy to prevent pertussis in early infancy. J Med Microbiol. 2018;67(10):1426-56.

25. Moher D, Liberati A, Tetzlaff J, et al. Preferred Reporting Items for Systematic Reviews and Meta-Analyses: The PRISMA Statement. Ann Intern Med. 2009; 151:264-9. https://doi.org/10.7326/0003-4819-151-4-200908180-00135.

26. Higgins JP, Altman DG, Gøtzsche PC, Jüni P, Moher D, Oxman AD, Savović J, Schulz KF, Weeks L, Sterne JA. The Cochrane Collaboration's tool for assessing risk of bias in randomised trials. BMJ. 2011;343:1-9(d5928).

27. Sterne JA, Hernán MA, Reeves BC, Savović J, Berkman ND, Viswanathan M, Carpenter JR. ROBINS-I: a tool for assessing risk of bias in non-randomised studies of interventions. BMJ. 2016;355:1-7(i4919).

28. Guyatt GH, Oxman AD, Schünemann HJ, Tugwell P, Knottnerus A. GRADE guidelines: a new series of articles in the journal of clinical epidemiology. Clin Epidemiol. 2011;64(4):380-2.

29. Schünemann HJ, Cuello C, Akl EA, Mustafa RA, Meerpohl JJ, Thayer K, Sterne J. GRADE guidelines: 18. How ROBINS-I and other tools to assess risk of bias in nonrandomized studies should be used to rate the certainty of a body of evidence. J Clin Epidemiol. 2019;111:105-14.

30. Munoz FM, Bond NH, Maccato M, Pinell P, Hammill HA, Swamy GK, Walter EB, Jackson LA, Englund JA, Edwards MS, et al. Safety and immunogenicity of tetanus diphtheria and Acellular pertussis (Tdap) immunization during pregnancy in mothers and infants: a randomized clinical trial. JAMA. 2014; 311(17):1760-9.

31. Hoang HTT, Leuridan E, Maertens K, Nguyen TD, Hens N, Vu NH, Caboré RN, Duong HT, Huygen K, Van Damme $P$, et al. Pertussis vaccination during pregnancy in Vietnam: results of a randomized controlled trial pertussis vaccination during pregnancy. Vaccine. 2016;34(1):151-9.

32. Halperin S, Langley J, Ye L, MacKinnon-Cameron D, Elsherif M, Allen V, Smith B, Halperin B, McNeil S, Vanderkooi O, et al. A Randomized Controlled Trial of the Safety and Immunogenicity of Tetanus, Diphtheria, and Acellular Pertussis Vaccine Immunization During Pregnancy and Subsequent Infant Immune Response. Clin Infect Dis. 2018;67(7):1063-71 2018.

33. Berenson AB, Hirth JM, Rahman M, Laz TH, Rupp RE, Sarpong KO. Maternal and infant outcomes among women vaccinated against pertussis during pregnancy. Human Immunotherapeutics. 2016;12(8):1965-71.

34. DeSilva M, Vazquez-Benitez G, Nordin JD, Lipkind HS, Romitti PA, DeStefano F, Kharbanda EO. Tdap vaccination during pregnancy and microcephaly and other structural birth defects in offspring. JAMA. 2016;316(17):1823-5.

35. DeSilva M, Vazquez-Benitez G, Nordin JD, Lipkind HS, Klein NP, Cheetham TC, Naleway AL, Hambidge SJ, Lee GM, Jackson ML, et al. Maternal Tdap vaccination and risk of infant morbidity. Vaccine. 2017;35(29):3655-60.

36. Donegan KKBBP. Safety of pertussis vaccination in pregnant women in UK: observational study. BMJ. 2014;349:1-6.

37. Griffin JB, Yu L, Watson D, Turner N, Walls T, Howe AS, Jiang Y, PetousisHarris $\mathrm{H}$. Pertussis immunisation in pregnancy safety (PIPS) study: a retrospective cohort study of safety outcomes in pregnant women vaccinated with Tdap vaccine. Vaccine. 2018;36(34):5173-9.

38. Kharbanda EO, Vazquez-Benitez G, Lipkind HS, et al. Evaluation of the association of maternal pertussis vaccination with obstetric events and birth outcomes. JAMA. 2014;312(18):1897-904.

39. Kharbanda EO, Vazquez-Benitez G, Lipkind HS, Klein NP, Cheetham TC, Naleway AL, Lee GM, Hambidge S, Jackson ML, Omer SB, et al. Maternal Tdap vaccination: coverage and acute safety outcomes in the vaccine safety datalink, 2007-2013. Vaccine. 2016;34(7):968-73.

40. Layton JB, Butler AM, Li D, Boggess KA, Weber DJ, McGrath LJ, Becker-Dreps S. Prenatal Tdap immunization and risk of maternal and newborn adverse events. Vaccine. 2017;35(33):4072-8.

41. Morgan JL, Baggari SR, Mclntire DD, Sheffield JS. Pregnancy Outcomes After Antepartum Tetanus, Diphtheria, and Acellular Pertussis Vaccination. Obstet Gynecol. 2015;125(6):1433-38.

42. Shakib JH, Korgenski K, Sheng X, Varner MW, Pavia AT, Byington CL. Tetanus, Diphtheria, Acellular Pertussis Vaccine during Pregnancy: Pregnancy and Infant Health Outcomes. J Pediatr. 2013;163(5):1422-6 e1424.

43. Amirthalingam G, Andrews N, Campbell H, Ribeiro S, Kara E, Donegan K, Fry NK, Miller E, Ramsay M. Effectiveness of maternal pertussis vaccination in England: an observational study. Lancet. 2014;384(9953):1521-8.

44. Baxter R, Bartlett J, Fireman B, Lewis E, Klein NP. Effectiveness of vaccination during pregnancy to prevent infant pertussis. Pediatrics. 2017;139(5):1-8.

45. Becker-Dreps S, Butler AM, McGrath LJ, Boggess KA, Weber DJ, Li D, Hudgens MG, Layton JB. Effectiveness of prenatal tetanus, diphtheria, Acellular pertussis vaccination in the prevention of infant pertussis in the $U$. S. Am J Prev Med. 2018;55(2):159-66.

46. Bellido-Blasco J, Guiral-Rodrigo S, Miguez-Santiyan A, Salazar-Cifre A, Gonzalez-Moran F. A case-control study to assess the effectiveness of pertussis vaccination during pregnancy on newborns, Valencian community, Spain, 1 march 2015 to 29 February 2016. Euro Surveill. 2017;22(22):1-7.

47. Dabrera G, Amirthalingam G, Andrews N, Campbell H, Ribeiro S, Kara E, Fry NK, Ramsay M. A case-control study to estimate the effectiveness of maternal pertussis vaccination in protecting newborn infants in England and Wales, 2012-2013. Clin Infect Dis. 2014;60(3):333-7.

48. Saul N, Wang K, Bag S, Baldwin H, Alexander K, Chandra M, Thomas J, Quinn H, Sheppeard V, Conaty S. Effectiveness of maternal pertussis vaccination in preventing infection and disease in infants: the NSW public health network case-control study. Vaccine. 2018;36(14):1887-92.

49. Skoff TH, Blain AE, Watt J, Scherzinger K, McMahon M, Zansky SM, Kudish K, Cieslak PR, Lewis M, Shang N, et al. Impact of the US maternal tetanus, diphtheria, and Acellular pertussis vaccination program on preventing pertussis in infants <2 months of age: a case-control evaluation. Clin Infect Dis. 2017;65(12):1977-83.

50. Kharbanda EO, Vazquez-Benitez G, Lipkind HS, Klein NP, Cheetham TC, Naleway AL, Lee GM, Hambidge S, Jackson ML, Omer SB, et al. Maternal Tdap vaccination: Coverage and acute safety outcomes in the vaccine safety datalink, 2007-2013. Vaccine. 2016;34(7):968-73.

51. Villarreal Pérez JZ, Ramírez Aranda JM, de la O Cavazos M, MdJ ZO, Perales Dávila J, Ballesteros Elizondo MR, Gómez Meza MV, García Elizondo FJ, Rodríguez González AM. Randomized clinical trial of the safety and immunogenicity of the Tdap vaccine in pregnant Mexican women. Human Vaccin Immunotherapeutics. 2017;13(1):128-35.

52. Maertens K, Caboré RN, Huygen K, Vermeiren S, Hens N, Van Damme P, Leuridan E. Pertussis vaccination during pregnancy in Belgium: Follow-up of 
infants until 1 month after the fourth infant pertussis vaccination at 15 months of age. Vaccine. 2016;34(31):3613-9.

53. Wanlapakorn N, Maertens $K$, Chaithongwongwatthana S, Srimuan $D_{\text {, }}$ Suratannon N, Vongpunsawad S, Mai Phuong Tran T, Hens N, Van Damme $\mathrm{P}$, Locht C, et al. Assessing the reactogenicity of Tdap vaccine administered during pregnancy and antibodies to Bordetella pertussis antigens in maternal and cord sera of Thai women. Vaccine. 2018;36(11):1453-9.

54. Layton JB, Butler AM, Li D, Boggess KA, Weber DJ, LJ MG, Becker-Dreps S. Prenatal Tdap immunization and risk of maternal and newborn adverse events. Vaccine. 2017;35(33):4072-78.

55. Bonhoeffer J, Kochhar S, Hirschfeld S, Heath PT, Jones CE, Bauwens J, Honrado Á, Heininger U, Muñoz FM, Eckert L, et al. Global alignment of immunization safety assessment in pregnancy - the GAIA project. Vaccine. 2016;34(49):5993-7.

56. Fortner KB, Swamy GK, Broder KR, Jimenez-Truque N, Zhu Y, Moro PL, Liang J, Walter EB, Heine RP, Moody MA, et al. Reactogenicity and immunogenicity of tetanus toxoid, reduced diphtheria toxoid, and acellular pertussis vaccine (Tdap) in pregnant and nonpregnant women. Vaccine. 2018;36(42):6354-60.

57. Fortner KB, Edwards KM, Broder KR, Jimenez N, Zhu Y, Walter EB, Heine RP, Moro P, Liang JL, Swamy GK. Reactogenicity of tetanus toxoid, reduced diphtheria toxoid, and acellular pertussis vaccine (Tdap) in pregnant women. Am J Obstet Gynecol. 2016;214(1):S193-4.

58. Oh K, Kim S, Hong J, Maymon E, Erez O, Panaitescu B, Gomez-Lopez N, Romero R, Hyun B, Yoon B. Twenty-four percent of patients with clinical chorioamnionitis in preterm gestations have no evidence of either cultureproven intraamniotic infection or intraamniotic inflammation. Am J Obstet Gynecol. 2017;216(6):604-e601.

59. Tita A, Andrews W. Diagnosis and management of clinical chorioamnionitis. Clin Perinatol. 2010;37(2):339-54.

60. Kramer B, Garnier Y. Chorioamnionitis-Eine inflammatorische Multiorganerkrankung des Fetus? Geburtshilfe Frauenheilkd. 2007;67(06): 602-10.

61. Mor G, Aldo P, Alvero AB. The unique immunological and microbial aspects of pregnancy. Nat Rev Immunol. 2017;17(8):469.

62. Abramovici A, Szychowski JM, Biggio JR, Sakawi Y, Andrews WW, Tita AT Epidural use and clinical chorioamnionitis among women who delivered vaginally. Am J Perinatol. 2014;31(11):1009-14.

63. Riley $L E$, Celi AC, Onderdonk AB, Roberts DJ, Johnson LC, Tsen LC, Leffert $L$, Pian-Smith MCM, Heffner $\amalg$, Haas ST, et al. Association of Epidural-Related Fever and Noninfectious Inflammation in term labor. Obstet Gynecol. 2011; 117(3):588-95.

64. Lieberman $\mathrm{E}$, O'donoghue C. Unintended effects of epidural analgesia during labor: a systematic review. Am J Obstet Gynecol. 2002;186(5):31-68

65. Centers for Disease Control and Prevention (CDC). Updated recommendations for use of tetanus toxoid, reduced diphtheria toxoid, and acellular pertussis vaccine (Tdap) in pregnant women--Advisory Committee on Immunization Practices (ACIP), 2012. Morb Mortal Wkly Rep. 2013;62(7): $131-5$.

66. Amirthalingam G, Letley L, Campbell H, Green D, Yarwood J, Ramsay M. Lessons learnt from the implementation of maternal immunization programs in England. Hum Vaccin Immunother. 2016;12(11):2934-9.

\section{Publisher's Note}

Springer Nature remains neutral with regard to jurisdictional claims in published maps and institutional affiliations.

Ready to submit your research? Choose BMC and benefit from:
- fast, convenient online submission
- thorough peer review by experienced researchers in your field
- rapid publication on acceptance
- support for research data, including large and complex data types
- gold Open Access which fosters wider collaboration and increased citations
- maximum visibility for your research: over 100M website views per year
At BMC, research is always in progress.
Learn more biomedcentral.com/submissions

